\title{
A REVIEW OF SELECTED TOPICS IN MAJORIZATION THEORY
}

\author{
MAREK NIEZGODA \\ Department of Applied Mathematics and Computer Science \\ University of Life Sciences in Lublin \\ Akademicka 13, 20-950 Lublin, Poland \\ E-mail: marek.niezgoda@up.lublin.pl
}

In memory of Professor Ky Fan

\begin{abstract}
In this expository paper, some recent developments in majorization theory are reviewed. Selected topics on group majorizations, group-induced cone orderings, Eaton triples, normal decomposition systems and similarly separable vectors are discussed. Special attention is devoted to majorization inequalities. A unified approach is presented for proving majorization relations for eigenvalues and singular values of matrices. Some methods based on the Chebyshev functional and similarly separable vectors are described. Generalizations of Hardy-LittlewoodPólya Theorem and Schur-Ostrowski Theorem are presented. Generalized Schur-convex functions are investigated. Extensions of Ky Fan inequalities are provided. Applications to Grüss and Ostrowski type inequalities are given.
\end{abstract}

1. Introduction and summary. The theory of majorization has many various applications in a number of fields, including matrix theory, convex analysis, probability, statistics, geometry, Lie theory, numerical analysis, optimization, etc. (see [2, 3, 4, 15, 16. 17, 18, 35, 44, 45, 51, 52, 70, 171]). The interested reader may consult the book Inequalities: Theory of Majorization and its Applications by W. A. Marshall, I. Olkin and B. C. Arnold [39] for the richness of applications in diverse disciplines (see also [5, 6, 27]).

In the literature, special attention is paid to majorization inequalities for linear operators (see [2, 3, 4, 45, 51, 52, 42, 69]). The importance of such results lies in the fact

2010 Mathematics Subject Classification: Primary 06F20, 15A42, 15A30, 15A21; Secondary 26A51, 26D10, 26D15, 39B62.

Key words and phrases: majorization, Schur-convex function, group majorization, group-induced cone ordering, Eaton triple, normal decomposition system, Schur inequality, eigenvalue, singular value, Chebyshev functional, similarly separable vectors, Chebyshev type inequality, Ky Fan type inequality, Grüss type inequality, Ostrowski type inequality.

The paper is in final form and no version of it will be published elsewhere. 
that they are related to eigenvalues and singular values of the operators. On the other hand, many existing results depend on the positivity of certain functionals. The aim of this survey article is to demonstrate two general methods for generalizing some classical majorization results. The first is based on the theory of Eaton triples and the second relies on the generalized Chebyshev functional and similarly separable vectors.

In this expository paper, we review some recent developments in the majorization theory. We focus on group majorizations, group-induced cone orderings, normal decomposition systems (ND systems), Eaton triples (E-systems) and similarly separable vectors. We quote the relevant material mainly from [48, 50, 51, 54, 55, 57, 56, 58, 59, 60.

The outline of the paper is as follows. In Section 2 we collect some basic facts from group majorization theory (see [15, 16, 18, 24, 34, 35, 42, 45, 46, 68, 70, 71]). The results are presented in Sections 38 .

In Section 3 we offer a unified approach to the problem of establishing majorization inequalities concerning eigenvalues and singular values of matrices [45, 47, 51, 52, 53. In particular, we show a relationship between such inequalities and decomposition statements for matrices as Spectral Decomposition, Singular Value Decomposition Theorem, Autonne Decomposition and Takagi Decomposition [45, 51.

Section 4 contains a discussion of morphisms of E-systems. Firstly, the motivation for this notion is provided. Namely, morphisms are characterized by a $G$-majorization inequality of the mentioned type. Secondly, a method for constructing morphisms is pointed out via simple morphisms. Lastly, homomorphisms are employed to give a technique of construction of Eaton triples.

Section 5 is devoted to the Chebyshev functional [50, 61]. By making use of similarly separable vectors, some sufficient and necessary conditions are provided for the functional to be nonnegative [48, 50, 57, 60]. The class of separable vectors on $\mathbb{R}^{n}$ includes many important subclasses such as monotone, monotone in mean, star-shaped, convex $n$-tuples, etc.

In Section 6 the similarity method is utilized to show some generalizations of HardyLittlewood-Pólya Theorem and of Schur-Ostrowski Theorem [57, 59]. Additionally, extended group majorization is studied.

Further applications are given in Section 7. Here Shi type inequalities are investigated [58. As corollaries, some $G$-majorization extensions of Ky Fan inequality are presented.

Finally, in Section 8, a new approach to Grüss and Ostrowski type inequalities is shown [57. By replacing the standard bounding constants by some corresponding bounding functions, some tighter estimates can be provided.

\section{Preliminaries}

2.1. Majorization and Schur-convex functions. We begin with some notation and terminology.

The decreasing rearrangement of a vector $z=\left(z_{1}, z_{2}, \ldots, z_{n}\right) \in \mathbb{R}^{n}$ is defined by

$$
z_{\downarrow}=\left(z_{[1]}, z_{[2]}, \ldots, z_{[n]}\right),
$$

where $z_{[i]}$ denotes the $i$ th largest entry of $z, i=1,2, \ldots, n$. Thus $z_{[1]} \geq z_{[2]} \geq \ldots \geq z_{[n]}$ are the entries of $z$ in decreasing order. 
Definition 2.1 (Weak majorization). A vector $x=\left(x_{1}, \ldots, x_{n}\right) \in \mathbb{R}^{n}$ is said to be weakly majorized by a vector $y=\left(y_{1}, \ldots, y_{n}\right) \in \mathbb{R}^{n}$, in symbols $x \prec_{w} y$, if

$$
\sum_{i=1}^{k} x_{[i]} \leq \sum_{i=1}^{k} y_{[i]} \quad \text { for } k=1,2, \ldots, n .
$$

The preorder $\prec_{w}$ on $\mathbb{R}^{n}$ is called weak majorization [39, p. 12].

Definition 2.2 (Majorization). A vector $x=\left(x_{1}, \ldots, x_{n}\right) \in \mathbb{R}^{n}$ is said to be majorized by a vector $y=\left(y_{1}, \ldots, y_{n}\right) \in \mathbb{R}^{n}$, in symbols $x \prec y$, if (1) holds and, in addition,

$$
\sum_{i=1}^{n} x_{[i]}=\sum_{i=1}^{n} y_{[i]} .
$$

The preorder $\prec$ on $\mathbb{R}^{n}$ is called majorization [39, p. 8].

Below we review some basic properties of the majorization preorder $\prec$. Thus we give motivation for the notion of group-induced cone ordering defined and described in the next subsection.

A geometric interpretation of majorization is as follows.

Theorem $2.3(\underline{64})$. Let $x, y \in \mathbb{R}^{n}$. Then

$$
x \prec y \quad \text { iff } \quad x \in \operatorname{conv} \mathbb{P}_{n} y,
$$

where $\mathbb{P}_{n}$ is the permutation group acting on $\mathbb{R}^{n}$, and conv $\mathbb{P}_{n} y$ stands for the convex hull of the set $\mathbb{P}_{n} y=\left\{p y: p \in \mathbb{P}_{n}\right\}$.

It is easily seen that the set $D=\left(\mathbb{R}^{n}\right)_{\downarrow}=\left\{z_{\downarrow}: z \in \mathbb{R}^{n}\right\}$ is a convex cone in $\mathbb{R}^{n}$. The following two properties (A1)-(A2) are met:

(A1) $D \cap \mathbb{P}_{n} z$ is not empty for each $z \in \mathbb{R}^{n}$, i.e., $\mathbb{R}^{n}=\bigcup_{p \in \mathbb{P}_{n}} p D$,

(A2) the rearrangement inequality holds:

$$
\langle x, p y\rangle \leq\langle x, y\rangle \quad \text { for } x, y \in D \text { and } p \in \mathbb{P}_{n},
$$

where $\langle\cdot, \cdot\rangle$ means the standard inner product on $\mathbb{R}^{n}$.

Condition (A1) means that each $z \in \mathbb{R}^{n}$ has the decomposition

$$
z=p z_{\downarrow} \quad \text { for some } p \in \mathbb{P}_{n} .
$$

On the other hand, by employing the standard norm $\|z\|=\langle z, z\rangle^{1 / 2}$ for $z \in \mathbb{R}^{n}$, condition (A2) can be restated as

$$
\|x-y\| \leq\|x-p y\| \quad \text { for } x, y \in D \text { and } p \in \mathbb{P}_{n},
$$

or, equivalently,

$$
\|x-y\|=\min _{p \in \mathbb{P}_{n}}\|x-p y\| \quad \text { for } x, y \in D .
$$

Properties of majorization $\prec$ depend on the geometry of the cone $D$ and its dual cone

$$
\text { dual } D=\left\{w \in \mathbb{R}^{n}:\langle w, z\rangle \geq 0 \quad \text { for } z \in D\right\} .
$$


For example, for $z \in D$ one has $z_{\downarrow}=z$ and $z_{[i]}=z_{i}, i=1,2, \ldots, n$. Therefore, if $x, y \in D$ then Definition 2.2 simplifies to

$$
x \prec y \quad \text { iff } \quad \sum_{i=1}^{k} x_{i} \leq \sum_{i=1}^{k} y_{i} \quad \text { for } k=1,2, \ldots, n, \quad \text { and } \quad \sum_{i=1}^{n} x_{i}=\sum_{i=1}^{n} y_{i} .
$$

In other words, for $x, y \in D$,

$$
x \prec y \quad \text { iff } \quad\left\langle x, s_{k}\right\rangle \leq\left\langle y, s_{k}\right\rangle \quad \text { for } k=1,2, \ldots, n, n+1,
$$

where

$$
s_{k}=(\underbrace{1, \ldots, 1}_{k \text { times }}, \underbrace{0, \ldots, 0}_{n-k \text { times }}) \quad \text { for } k=1,2, \ldots, n, \quad \text { and } \quad s_{n+1}=-s_{n}=(\underbrace{-1, \ldots,-1}_{n \text { times }})
$$

are the vectors spanning the convex cone $D$, i.e.,

$$
D=\text { cone }\left\{s_{k}: k=1,2, \ldots, n, n+1\right\} .
$$

Therefore the majorization preorder $\prec$, restricted to the convex cone $D$, is a cone preorder induced by the dual cone of $D$, that is, for $x, y \in D$,

$$
x \prec y \quad \text { iff } \quad y-x \in \text { dual } D .
$$

Here

$$
\begin{gathered}
\text { dual } D=\text { cone }\left\{r_{k}: k=1,2, \ldots, n-1\right\}, \\
r_{k}=(\underbrace{0, \ldots, 0}_{k-1 \text { times }}, 1,-1, \underbrace{0, \ldots, 0}_{n-k-1 \text { times }}) \text { for } k=1,2, \ldots, n-1 .
\end{gathered}
$$

The above symbol cone $A$ denotes the convex cone of all nonnegative finite linear combinations of vectors in subset $A$ of a linear space.

Definition 2.4 (Schur-convexity, Schur-concavity). Let $A \subset \mathbb{R}^{n}$ be a (nonempty) symmetric set (i.e., $p x \in A$ whenever $x \in A$ and $p \in \mathbb{P}_{n}$ ).

A function $f: A \rightarrow \mathbb{R}$ is said to be Schur-convex (resp. Schur-concave) on $A$, if for $x, y \in A$,

$$
x \prec y \quad \text { implies } \quad f(x) \leq(\geq) f(y) .
$$

Schur-convex (resp. Schur-concave) functions on $A=\mathbb{R}^{n}$ are simply called Schurconvex (resp. Schur-concave). For abbreviation, Schur-convex (resp. Schur-concave) functions are called $S$-convex (resp. S-concave). i.e.,

It is well-known that a Schur-convex function $f: A \rightarrow \mathbb{R}$ is necessarily symmetric,

$$
f(p x)=f(x) \quad \text { for } x \in A \text { and } p \in \mathbb{P}_{n} .
$$

2.2. $G$-majorization and group-induced cone orderings. Throughout $(V,\langle\cdot, \cdot\rangle)$ is a finite-dimensional real inner product space. The symbol $O(V)$ stands for the orthogonal group acting on $V$.

Definition 2.5 (Group majorization). Let $G$ be a subgroup of $O(V)$. Given vectors $x, y \in V$, we write $x \prec_{G} y$ if $x$ lies in the convex hull conv $G y$ of the $G$-orbit $G y=\{g y$ : $g \in G\}$, i.e.,

$$
x \prec_{G} y \quad \text { iff } \quad x \in \operatorname{conv} G y .
$$


The preorder $\prec_{G}$ is called group majorization with respect to $G$, in short, $G$-majorization (see [15], [39, p. 589]).

For instance, if $G=\mathbb{P}_{n}$ is the group of $n \times n$ permutation matrices acting on $V=\mathbb{R}^{n}$, then $\prec_{G}$ is the majorization preorder $\prec$ on $\mathbb{R}^{n}$ [39, p. 10, p. 34, p. 162].

If $x \prec_{G} y$ and $y \prec_{G} x$ then we write $x \equiv_{G} y$.

The $G$-majorization $\prec_{G}$ is $G$-invariant on $V$ in the sense that for any $x, y \in V$,

$$
x \prec_{G} y \quad \text { iff } \quad g_{1} x \prec_{G} g_{2} y \quad \text { for } g_{1}, g_{2} \in G .
$$

Definition 2.6 (GIC ordering). $G$-majorization $\prec_{G}$ induced by a compact group $G \subset$ $O(V)$ is said to be a group-induced cone ordering (in short, GIC ordering) if there exists a closed convex cone $D \subset V$ such that

(A1) $D \cap G z$ is not empty for each $z \in V$,

(A2) $\langle x, g y\rangle \leq\langle x, y\rangle$ for $x, y \in D$ and $g \in G$.

Any GIC ordering $\prec_{G}$, restricted to its convex cone $D$, is the cone ordering on $D$ induced by $C=$ dual $D$. That is, for $x, y \in D$, the following statements are equivalent:

(i) $x \prec_{G} y$,

(ii) $\langle y-x, s\rangle \geq 0$ for $s \in D$,

(iii) $\left\langle y-x, s_{i}\right\rangle \geq 0$ for $i=1, \ldots, k$, provided $D=$ cone $\left\{s_{1}, s_{2}, \ldots, s_{k}\right\}$.

Definition 2.7 ( $G$-increasing function). Let $A \subset V$ be a $G$-invariant set, i.e., $g x \in A$ whenever $x \in A$ and $g \in G$.

A function $f: A \rightarrow \mathbb{R}$ is said to be $G$-increasing on $A$, if for $x, y \in A$,

$$
x \prec_{G} y \quad \text { implies } \quad f(x) \leq f(y) .
$$

$G$-increasing functions $f: V \rightarrow \mathbb{R}$ on $A=V$ are simply called $G$-increasing.

Definition 2.8 ( $G$-invariant function). A function $f: A \rightarrow \mathbb{R}$ is said to be $G$-invariant, if $A$ is $G$-invariant and

$$
f(g x)=f(x) \quad \text { for } g \in G \text { and } x \in A .
$$

Each $G$-increasing function is necessarily $G$-invariant.

2.3. Normal decomposition systems and Eaton triples. Let $(V,\langle\cdot, \cdot\rangle)$ be a finitedimensional real inner product space and let $G \subset O(V)$ be a closed group acting on $V$.

If $\prec_{G}$ is a GIC ordering then for each $x \in V$ the intersection $D \cap G x$ is a singleton set consisting of the unique vector denoted by $x_{\downarrow}$ [45, p. 14].

Definition 2.9 (Normal map). If axioms (A1)-(A2) hold for some closed convex cone $D \subset V$, then the map

$$
(\cdot)_{\downarrow}: V \ni x \rightarrow x_{\downarrow} \in D,
$$

where $\left\{x_{\downarrow}\right\}=D \cap G x$, is called normal map.

The map $(\cdot)_{\downarrow}$ is $G$-invariant and idempotent. Its range is the convex cone $D$. The restriction of $(\cdot)_{\downarrow}$ to $D$ is the identity. For each $x \in V$, the vectors $x_{\downarrow}$ and $x$ are equivalent in the sense that $x_{\downarrow} \prec_{G} x$ and $x \prec_{G} x_{\downarrow}$. 
Under axioms (A1)-(A2), for $x, y \in V$ we have

$$
\begin{gathered}
y \prec_{G} x \quad \text { iff } \quad y_{\downarrow} \prec_{G} x_{\downarrow} \quad \text { iff } \quad\left\langle z, y_{\downarrow}\right\rangle \leq\left\langle z, x_{\downarrow}\right\rangle \text { for } \quad z \in D, \\
y \prec_{G} x \quad \text { iff } \quad\langle z, g y\rangle \leq\left\langle z, x_{\downarrow}\right\rangle \text { for } z \in D \text { and } g \in G .
\end{gathered}
$$

In light of (A1)-(A2), each vector $x$ in $V$ has its canonical (normal) decomposition:

$$
x=g x_{\downarrow} \quad \text { for some } g \in G .
$$

Definition 2.10 (Normal decomposition system). If axioms (A1)-(A2) hold for closed convex cone $D \subset V$ then the triple $\left(V, G,(\cdot)_{\downarrow}\right)$ is called a normal decomposition system (in short, ND system) [34, 35].

Definition 2.11 (Eaton triple). If axioms (A1)-(A2) hold for closed convex cone $D \subset V$ then the triple $(V, G, D)$ is called an Eaton triple (in short, E-system) [15, 16, 70].

The above-mentioned notions play a unifying role in statistics, matrix theory, Lie theory, etc. (see [15, 18, 34, 35, 42, 47, 70]).

2.4. Examples of Eaton triples. An important class of examples is provided by finite reflection groups according to the following definition and theorem.

DeFinition 2.12 (Reflection group). A group $G \subset O(V)$ is said to be a reflection group if $G$ is the closure of a subgroup of $O(V)$ generated by some set of the reflections

$$
S_{r} x=x-2 \frac{\langle x, r\rangle}{\langle r, r\rangle} r \quad \text { for } x \in V,
$$

where $0 \neq r \in V[18$, 30].

Theorem 2.13 ([18, Lemma 4.1], [68, Theorem 4.1]). Let $G \subset O(V)$ be a finite group. Then the following three statements are equivalent:

(i) $G$ is a finite reflection group.

(ii) The G-majorization $\prec_{G}$ is a GIC ordering for some closed convex cone $D \subset V$.

(iii) The triple $(V, G, D)$ is an Eaton triple for some closed convex cone $D \subset V$.

We need some notation (see [51]).

$\mathbb{R}_{+}^{n}=$ the convex cone of nonnegative vectors in $\mathbb{R}^{n}$,

$\mathbb{R}_{\downarrow}^{n}=$ the convex cone of nonincreasing vectors in $\mathbb{R}^{n}$,

$\mathbb{R}_{+\downarrow}^{n}=$ the convex cone of nonnegative nonincreasing vectors in $\mathbb{R}^{n}$,

$\mathbb{O}_{n}=$ the group of $n \times n$ real orthogonal matrices,

$\mathbb{D O}_{n}=$ the group of $n \times n$ diagonal orthogonal matrices,

$\mathbb{P}_{n}=$ the group of $n \times n$ permutation matrices,

$\mathbb{G P}_{n}(\mathbb{R})=$ the group of $n \times n$ generalized permutation matrices, i.e., real matrices with exactly one nonzero entry with absolute value 1 in each row and column.

EXAmple 2.14 ([15, p. 16]). It is easily seen that $(V, G, D)$ is an Eaton triple for $V=\mathbb{R}^{n}$, $G=\mathbb{D O}_{n}$ and $D=\mathbb{R}_{+}^{n}$. Moreover, $G$ is a finite reflection group, and $y \prec_{G} x$ means $|y| \leq|x|$ for $x, y \in \mathbb{R}^{n}$, where $x_{\downarrow}=|x|=\left(\left|x_{1}\right|, \ldots,\left|x_{n}\right|\right)^{T}$.

EXAMPLE 2.15 ([15, p. 16]). It is known that if $V=\mathbb{R}^{n}, G=\mathbb{P}_{n}$ and $D=\mathbb{R}_{\downarrow}^{n}$, then $(V, G, D)$ is an Eaton triple, $G$ is a finite reflection group, and $y \prec_{G} x$ means $y \prec x$ for $x, y \in \mathbb{R}^{n}$. Furthermore, $x_{\downarrow}=\left(x_{[1]}, \ldots, x_{[n]}\right)^{T}$. 
ExAmple 2.16 ([15, p. 16]). Replacing $V, G$, and $D$ with $\mathbb{R}^{n}, \mathbb{G P}_{n}(\mathbb{R})$ and $\mathbb{R}_{+\downarrow}^{n}$, respectively, one obtains Eaton triple $(V, G, D)$ with finite reflection group $G$. Here $y \prec_{G} x$ reduces to $|y| \prec_{w}|x|$ for $x, y \in \mathbb{R}^{n}$, and, in addition, $x_{\downarrow}=\left(|x|_{[1]}, \ldots,|x|_{[n]}\right)^{T}$.

In forthcoming matrix examples, we need further notation (for the field $\mathbb{F}=\mathbb{C}$ or $\mathbb{R}$ ). $\mathbb{M}_{n}(\mathbb{F})=$ the vector space of $n \times n$ matrices over $\mathbb{F}$,

$\mathbb{H}_{n}=$ the (real) vector space of $n \times n$ Hermitian matrices,

$\mathbb{S}_{n}(\mathbb{F})=$ the vector space of $n \times n$ symmetric matrices over $\mathbb{F}$,

$\mathbb{K}_{n}(\mathbb{F})=$ the vector space of $n \times n$ skew-symmetric matrices over $\mathbb{F}$,

$\mathbb{D}_{n}(\mathbb{F})=$ the vector space of $n \times n$ diagonal matrices over $\mathbb{F}$,

$\mathbb{G P}_{n}(\mathbb{F})=$ the group of $n \times n$ generalized permutation matrices over $\mathbb{F}$, i.e., matrices with exactly one nonzero entry with magnitude 1 in each row and column,

$\mathbb{U}_{n}=$ the group of $n \times n$ unitary matrices,

$\mathbb{D} \mathbb{U}_{n}=$ the group of $n \times n$ diagonal unitary matrices,

$X^{*}=$ the conjugate transpose of matrix $X$,

$\lambda(X)=\left(\lambda_{1}(X), \lambda_{2}(X), \ldots, \lambda_{n}(X)\right)=$ the vector of eigenvalues of Hermitian matrix $X$ stated in nonincreasing order: $\lambda_{1}(X) \geq \lambda_{2}(X) \geq \ldots \geq \lambda_{n}(X)$,

$s(X)=\left(s_{1}(X), s_{2}(X), \ldots, s_{n}(X)\right)=$ the vector of singular values of matrix $X$, i.e., $s(X)=\lambda\left(X^{*} X\right)^{1 / 2}$ with $s_{1}(X) \geq s_{2}(X) \geq \ldots \geq s_{n}(X)$,

$d(X)=$ the vector of diagonal entries of matrix $X$,

$\operatorname{diag} z=$ the diagonal matrix with the entries of a vector $z \in \mathbb{R}^{n}$ on the main diagonal,

$\operatorname{Re} X=$ the real part of matrix $X=\left(x_{i, j}\right)$, i.e., $\operatorname{Re} X=\left(\operatorname{Re} x_{i, j}\right)$,

$U_{1}(\cdot) U_{2}=$ the matrix operator of the form $X \rightarrow U_{1} X U_{2}$, where $X, U_{1}$ and $U_{2}$ are matrices.

We now present two important examples of Eaton triples related to the eigenvalues of an Hermitian matrix and to the singular values of a complex matrix, respectively.

EXAmple 2.17 (see [15, p. 17], [35, pp. 943-944], [55, p. 619]). Consider $V=\mathbb{H}_{n}$ with the inner product defined by

$$
\langle X, Y\rangle=\operatorname{Retr} X Y \quad \text { for } X, Y \in \mathbb{H}_{n} .
$$

Let $G$ be the group of all linear operators of the form

$$
X \rightarrow U X U^{*} \quad \text { for } X \in \mathbb{H}_{n} \text { with } U \in \mathbb{U}_{n} .
$$

Then $(V, G, D)$ is an E-system for

$$
D=\left\{\operatorname{diag}\left(z_{1}, \ldots, z_{n}\right) \in \mathbb{D}_{n}: z_{1} \geq \ldots \geq z_{n}\right\} .
$$

Indeed, axiom (A1) reduces to the Spectral Theorem, and axiom (A2) becomes the FanTheobald's trace inequality [19, 72]. Furthermore, we have

$$
\begin{gathered}
X_{\downarrow}=\operatorname{diag} \lambda(X) \text { for } X \in \mathbb{H}_{n}, \\
Y \prec_{G} X \text { iff } \lambda(Y) \prec \lambda(X) \text { for } X, Y \in \mathbb{H}_{n}
\end{gathered}
$$

(see $[2]$ and [15, p. 17]). In consequence, $\prec_{G}$ on $\mathbb{D}_{n}(\mathbb{R})$ may be identified with the classical majorization $\prec$ on $\mathbb{R}^{n}$.

Schur's inequality ([66, [39, p. 300]) for an $n \times n$ Hermitian matrix $X$ says that

$$
d(X) \prec \lambda(X) .
$$


ExAmple 2.18 (see [15, pp. 17-18], [35, pp. 944-945], [55, p. 619]). Take $V=\mathbb{M}_{n}(\mathbb{C})$ with real inner product given by

$$
\langle X, Y\rangle=\operatorname{Retr} X Y^{*} \quad \text { for } X, Y \in \mathbb{M}_{n}(\mathbb{C}),
$$

and let $G$ be the group of all linear operators of the form

$$
X \rightarrow U_{1} X U_{2} \quad \text { for } X \in \mathbb{M}_{n}(\mathbb{C}), \text { where } U_{1}, U_{2} \in \mathbb{U}_{n} .
$$

Put

$$
D=\left\{\operatorname{diag}\left(z_{1}, \ldots, z_{n}\right) \in \mathbb{D}_{n}: z_{1} \geq \ldots \geq z_{n} \geq 0\right\} .
$$

Here (A1) is the Singular Values Decomposition Theorem [39, p. 771], and (A2) is the trace inequality of von Neumann [39, p. 789]. So, $(V, G, D)$ is an E-system. In addition, we have

$$
\begin{gathered}
X_{\downarrow}=\operatorname{diag} s(X) \quad \text { for } X \in \mathbb{M}_{n}(\mathbb{C}), \\
Y \prec_{G} X \quad \text { iff } \quad s(Y) \prec_{w} s(X) \quad \text { for } X, Y \in \mathbb{M}_{n}(\mathbb{C})
\end{gathered}
$$

(see (2) and [15, pp. 17-18]).

It is well-known by Ky Fan's inequality [20] that for an $n \times n$ complex matrix $X$,

$$
d(X) \prec_{w} s(X) .
$$

3. $G$-majorization inequalities for linear operators. In this section we aim to show some majorization inequalities generated by certain linear operators. To this end we employ Eaton triples and normal maps. Such an approach gives a better understanding of many results on eigenvalues and singular values of certain classes of matrices.

As previously, it is assumed that $(V,\langle\cdot, \cdot\rangle)$ is an inner product space, $G$ is a closed subgroup of the orthogonal group $O(V)$, and $D \subset V$ is a closed convex cone.

3.1. G-majorization and orthoprojectors. We begin our discussion with $G$-majorization inequality (6) which generalizes, among others, Schur's inequality (3) and Ky Fan's inequality 4.

Theorem 3.1 ([51, Theorem 2.1]). Let $(V, G, D)$ be an Eaton triple. Assume $W$ is a linear subspace of $V, H$ is a subset of $G$, and $E$ is a subset of $D$. Let $P$ be the orthoprojector from $V$ onto $W$ and $Q$ be the orthoprojector from $\operatorname{span} D$ onto $\operatorname{span} E$.

$$
\text { If } Q D=E \text { and }
$$

$$
W=\bigcup_{h \in H} h E
$$

then

$$
P x \prec_{G} Q x_{\downarrow} \quad \text { for } \quad x \in V .
$$

Example 3.2. Let $V, G$ and $D$ be defined as in Example 2.17. Setting

$$
W=\mathbb{S}_{n}(\mathbb{R}), \quad H=\left\{U(\cdot) U^{T}: U \in \mathbb{O}_{n}\right\} \quad \text { and } \quad E=D
$$

(cf. [34, Example 7.4]), we obtain $P X=\operatorname{Re} X$ for $X \in \mathbb{H}_{n}$, and $Q$ is the identity on $\operatorname{span} D$. 
Condition (5) is fulfilled by Spectral Decomposition for matrices in $\mathbb{S}_{n}(\mathbb{R})$. From (6) we deduce that (cf. [2, p. 111])

$$
\lambda(\operatorname{Re} X) \prec \lambda(X) \quad \text { for } \quad X \in \mathbb{H}_{n} .
$$

Example 3.3. Assume $V, G$ and $D$ are defined as in Example 2.18. For $k \in\{1,2, \ldots, n\}$, let

$$
\begin{gathered}
W=\mathbb{M}_{k}(\mathbb{C}) \oplus 0_{n-k}, \quad H=\left\{U_{1}(\cdot) U_{2}: U_{1}, U_{2} \in \mathbb{U}_{k} \oplus I_{n-k}\right\}, \\
\text { and } \quad E=\left\{Z \in \mathbb{D}_{k}(\mathbb{R}) \oplus 0_{n-k}: z_{11} \geq \ldots \geq z_{k k} \geq 0\right\} .
\end{gathered}
$$

Condition (5) amounts to Singular Value Decomposition for matrices in $\mathbb{M}_{k}(\mathbb{C})$. The orthoprojector $P$ is given by

$$
P X=X_{11} \oplus 0_{n-k} \quad \text { for } \quad X \in \mathbb{M}_{n}(\mathbb{C}),
$$

where $X_{11}$ is the $k \times k$ principal submatrix of $X$. Additionally, $Q$ is the restriction of $P$ to $\mathbb{D}_{n}(\mathbb{R})$. Consequently, inequality (6) gives

$$
s\left(X_{11}\right) \prec_{w}\left(s_{1}(X), \ldots, s_{k}(X)\right) \quad \text { for } \quad X \in \mathbb{M}_{n}(\mathbb{C}) .
$$

EXAmPLE 3.4. Let $V=\mathbb{M}_{n}(\mathbb{R})$,

$$
\begin{aligned}
& G=\left\{U_{1}(\cdot) U_{2}: U_{1}, U_{2} \in \mathbb{O}_{n}\right\}, \quad D=\left\{Z \in \mathbb{D}_{n}(\mathbb{R}): d(Z) \in \mathbb{R}_{+\downarrow}^{n}\right\}, \quad W=\mathbb{K}_{n}(\mathbb{R}), \\
& H=\left\{U U_{0}(\cdot) U^{T}: U \in \mathbb{O}_{n}\right\} \quad \text { and } \quad E=\left\{s_{1} I_{2} \oplus \ldots \oplus s_{k} I_{2}: s_{1} \geq \ldots \geq s_{k} \geq 0\right\},
\end{aligned}
$$

where $n=2 k$ is even, $I_{2}=\left(\begin{array}{ll}1 & 0 \\ 0 & 1\end{array}\right), U_{0}$ is the $n \times n$ block-diagonal matrix $B \oplus \ldots \oplus B$ with $B=\left(\begin{array}{cc}0 & 1 \\ -1 & 0\end{array}\right)$. Condition [5] is satisfied by Autonne Decomposition (cf. [34, Example 7.5]).

It is clear that

$$
\begin{gathered}
P X=\frac{X-X^{T}}{2} \quad \text { for } \quad X \in \mathbb{M}_{n}(\mathbb{R}), \\
Q X=\frac{s_{1}+s_{2}}{2} I_{2} \oplus \ldots \oplus \frac{s_{n-1}+s_{n}}{2} I_{2} \quad \text { for } \quad X=\operatorname{diag}\left(s_{1}, \ldots, s_{n}\right) \in \mathbb{D}_{n}(\mathbb{R}) .
\end{gathered}
$$

By utilizing inequality (6) we obtain

$$
s\left(\frac{X-X^{T}}{2}\right) \prec_{w}\left(\frac{s_{1}+s_{2}}{2}, \frac{s_{1}+s_{2}}{2}, \ldots, \frac{s_{n-1}+s_{n}}{2}, \frac{s_{n-1}+s_{n}}{2}\right)
$$

for $X \in \mathbb{M}_{n}(\mathbb{R})$, where $s_{i}=s_{i}(X), i=1,2, \ldots, n$, (cf. [2, p. 109]).

3.2. Reduced triple of an Eaton triple. We introduce the notion of a reduced triple.

Definition 3.5 (Reduced triple). Given an Eaton triple $(V, G, D)$, set

$$
V_{0}=\operatorname{span} D \quad \text { and } \quad G_{0}=\left\{\left.g\right|_{V_{0}}: g \in G \quad \text { and } g V_{0}=V_{0}\right\} .
$$

If $\left(V_{0}, G_{0}, D\right)$ is an Eaton triple (under the inherited inner product), then it is called the reduced triple of $(V, G, D)$ [70].

It is important that if $\left(V_{0}, G_{0}, D\right)$ is the reduced triple of $(V, G, D)$ then $G_{0}$ is a finite reflection group acting on $V_{0}$ and the following reduction holds:

$$
y \prec_{G} x \quad \text { iff } \quad y_{\downarrow} \prec_{G} x_{\downarrow} \quad \text { iff } \quad y_{\downarrow} \prec_{G_{0}} x_{\downarrow} \quad \text { for } x, y \in V
$$

(see [45, Theorem 3.2], [18, Lemma 4.1, (35)], 68, Theorem 4.1]). 
Example 3.6. If $V, G, D$ are as in Example 2.17, then the reduced triple is $\left(V_{0}, G_{0}, D\right)$ for $V_{0}=\mathbb{D}_{n}(\mathbb{R}), G_{0}=\left\{S(\cdot) S^{T}: S \in \mathbb{P}_{n}\right\}$ and $D=\left\{Z \in \mathbb{D}_{n}(\mathbb{R}): d(Z) \in \mathbb{R}_{\downarrow}^{n}\right\}$. In consequence, $\left(V_{0}, G_{0}, D\right)$ can be identified with the E-system $\left(\mathbb{R}^{n}, \mathbb{P}_{n}, \mathbb{R}_{\downarrow}^{n}\right)$.

ExAmple 3.7. In the case of Example 2.18 the reduced triple $\left(V_{0}, G_{0}, D\right)$ of $(V, G, D)$ consists of $V_{0}=\mathbb{D}_{n}(\mathbb{R})$,

$$
G_{0}=\left\{C S(\cdot) S^{T}: S \in \mathbb{P}_{n}, C \in \mathbb{D O}_{n}\right\} \text { and } D=\left\{Z \in \mathbb{D}_{n}(\mathbb{R}): d(Z) \in \mathbb{R}_{+\downarrow}^{n}\right\} .
$$

Additionally, the reduced triple can be identified with the triple $\left(\mathbb{R}^{n}, \mathbb{G P}_{n}(\mathbb{R}), \mathbb{R}_{+\downarrow}^{n}\right)$.

Theorem 3.8 ([51, Theorem 2.7]). Let $(V, G, D)$ and $(W, H, E)$ be Eaton triples with reduced systems $\left(V_{0}, G_{0}, D\right)$ and $\left(W_{0}, H_{0}, E\right)$, respectively. Assume that $W \subset V, H \subset G$ and $D \subset E$. Let $P$ stand for the orthoprojector from $V$ onto $W$.

If $W_{0}=V_{0}$ then there exists a subset $\widehat{G} \subset G$ such that $W=\bigcup_{g \in \widehat{G}} g D$, and

$$
P x \prec_{G} x_{\downarrow} \quad \text { for } \quad x \in V \text {. }
$$

ExAmple 3.9. Let $V, G$ and $D$ be as in Example 2.18. Setting

$$
W=\mathbb{H}_{n}, \quad H=\left\{U(\cdot) U^{*}: U \in \mathbb{U}_{n}\right\} \quad \text { and } \quad E=\left\{Z \in \mathbb{D}_{n}(\mathbb{R}): d(Z) \in \mathbb{R}_{\downarrow}^{n}\right\}
$$

leads to

$$
P X=\frac{X+X^{*}}{2} \text { for } X \in \mathbb{M}_{n}(\mathbb{C}) .
$$

Application of Theorem 3.8 gives Fan-Hoffman's inequality (see [2, p. 109], [39, p. 327]):

$$
s\left(\frac{X+X^{*}}{2}\right) \prec_{w} s(X) \text { for } X \in \mathbb{M}_{n}(\mathbb{C}) .
$$

ExAmple 3.10. Letting $(V, G, D)$ to be as in Example 2.17 for $k \in\{1,2, \ldots, n\}$ we consider

$$
\begin{gathered}
W=\mathbb{H}_{k} \oplus \mathbb{H}_{n-k}, \quad H=\left\{U(\cdot) U^{*}: U \in \mathbb{U}_{k} \oplus \mathbb{U}_{n-k}\right\} \\
\text { and } \quad E=\left\{Z \in \mathbb{D}_{n}(\mathbb{R}): z_{11} \geq \ldots \geq z_{k k}, \quad z_{k+1 k+1} \geq \ldots \geq z_{n n}\right\} .
\end{gathered}
$$

Then we obtain

$$
P X=X_{11} \oplus X_{22} \quad \text { for } \quad X=\left(\begin{array}{ll}
X_{11} & X_{12} \\
X_{21} & X_{22}
\end{array}\right) \in \mathbb{H}_{n},
$$

where $X_{11}$ and $X_{22}$ are of sizes $k \times k$ and $(n-k) \times(n-k)$, respectively.

By making use of Theorem 3.8 we get Ky Fan's inequality (see [39, p. 308], cf. also [2. p. 97]):

$$
\left(\lambda\left(X_{11}\right), \lambda\left(X_{22}\right)\right) \prec \lambda(X) \quad \text { for } \quad X \in \mathbb{H}_{n} .
$$

3.3. Subsystems of E-systems. We are interested in the notion of a subsystem of an E-system and its role in deriving $G$-majorization inequalities.

Definition 3.11 (Subsystem of E-system). Let $(V, G, D)$ be an E-system. Assume $H$ is a closed subgroup of $G, W$ is an $H$-invariant subspace of $V$, and $E$ is a closed convex subcone of $D$. The triple $(W, H, E)$ is called a subsystem of $(V, G, D)$, if $(W, H, E)$ is an E-system (under the inherited inner product). For notation simplicity, we write $(W, H, E)$ in place of $\left(W,\left.H\right|_{W}, E\right)$ (cf. 34, 35]). 
A special class of subsystems is formed by the reduced triples. In Theorem 3.12 we characterize arbitrary subsystems of an E-system (cf. [45, Theorem 3.2]).

Theorem 3.12 ([51, Theorem 3.1]). Let $(V, G, D)$ be an E-system. Suppose $H$ is a closed subgroup of $G, W$ is an $H$-invariant subspace of $V$, and $E \subset W$ is a closed convex subcone of $D$. Let $P$ be the orthoprojector from $V$ onto $W$, and $Q$ be the orthoprojector from $\operatorname{span} D$ onto span $E$. Assume $Q D=E$.

Then the following statements are equivalent:

(a) $(W, H, E)$ is a subsystem of $(V, G, D)$.

(b) The following inequality holds

$$
P x \prec_{H} Q x_{\downarrow} \quad \text { for } x \in V .
$$

(c) The following inclusion holds

$$
W \subset \bigcup_{g \in G} g E,
$$

and, in addition, the orderings $\prec_{H}$ and $\prec_{G}$ coincide on $W$, i.e.

$$
y \prec_{H} x \quad \text { iff } \quad y \prec_{G} x \quad \text { for } \quad x, y \in W .
$$

(d) The following decomposition holds

$$
W=\bigcup_{h \in H} h E .
$$

ExAmPle 3.13. We take $V, G$ and $D$ to be as in Example 2.18, and we set

$$
W=\mathbb{D}_{n}(\mathbb{C}), \quad H=\left\{C S(\cdot) S^{T}: S \in \mathbb{P}_{n}, C \in \mathbb{D U}_{n}\right\} \quad \text { and } \quad E=D .
$$

Then (8) holds by Polar Decomposition for diagonal matrices. Moreover,

$$
P X=\operatorname{diag}\left(x_{11}, \ldots, x_{n n}\right) \quad \text { for } \quad X=\left(x_{i j}\right) \in \mathbb{M}_{n}(\mathbb{C}) .
$$

Applying (7) yields the following Ky Fan's result [39, p. 314]:

$$
\left(\left|x_{11}\right|, \ldots,\left|x_{n n}\right|\right) \prec_{w}\left(s_{1}(X), \ldots, s_{n}(X)\right) \quad \text { for } \quad X \in \mathbb{M}_{n}(\mathbb{C}) .
$$

3.4. G-doubly stochastic operators. Here we show that $G$-doubly stochastic operators induce some subsystems of E-systems.

Definition 3.14 ( $G$-doubly stochastic operator). Let $(V, G, D)$ be an E-system. A linear operator $L: V \rightarrow V$ is called $G$-doubly stochastic if

$$
L x \prec_{G} x \quad \text { for } \quad x \in V .
$$

It can be proved that $L$ is $G$-doubly stochastic iff so is its adjoint $L^{*}$ defined by

$$
\langle L x, y\rangle=\left\langle x, L^{*} y\right\rangle \quad \text { for all } x, y \in V .
$$

ExAmple 3.15. If $V=\mathbb{R}^{n}, G=\mathbb{P}_{n}$ and $D=\mathbb{R}_{\downarrow}^{n}$ as in Example 2.15, and if $L$ is an $n \times n$ matrix, then (9) amounts to $L x \prec x$ for $x \in \mathbb{R}^{n}$, which is equivalent to the conditions $L \geq 0, L v=v$ and $L^{T} v=v$, where $v=(1, \ldots, 1)^{T} \in \mathbb{R}^{n}$ (see [3, p. 169, Theorem 3.1]). In other words, $L$ is a matrix with nonnegative entries and with row and column sums equal to 1 . Such matrices $L$ are said to be doubly stochastic. Clearly, $L$ is doubly stochastic if and only if so is $L^{T}$. 
Given an E-system $(V, G, D)$, a point $x \in V$ is said to be regular provided $x \in V_{r}=$ $\bigcup_{g \in G} g$ ri $D$, where ri $(\cdot)$ denotes "the relative interior of".

The set $V_{r}$ is dense in $V$, i.e., $\operatorname{cl} V_{r}=V$, where $\mathrm{cl}(\cdot)$ means "the closure of".

Theorem 3.16 ([51, Theorem 4.1]). Suppose $(V, G, D)$ is an E-system. Let $W$ be a subspace of $V$ such that $D \subset W$, and let $H=\{h \in G: h D \subset W\}$. Then the following statements are equivalent:

(i) $W=\bigcup_{h \in H} h D$.

(ii) There exists a G-doubly stochastic operator $L: V \rightarrow V$ such that $W=\{x \in V$ : $L x=x\}$, and, in addition, the set $V_{r} \cap W$ is dense in $W$.

Under condition (i), the set $H$ is a group if and only if $H=\{h \in G: h W=W\}$. In this case, the triple $(W, H, D)$ is a subsystem of $(V, G, D)$.

In some situations, the set $H$ in Theorem 3.16 can be replaced by a set $H_{0} \subset H$.

Example 3.17. Define $V, G$ and $D$ as in Example 2.18. Set $W=\mathbb{S}_{n}(\mathbb{C})$. Let $H$ be defined as in Theorem 3.16, and let $H_{0}=\left\{h_{0}=U(\cdot) U^{T}: U \in \mathbb{U}_{n}\right\}$. Then $W=\bigcup_{h_{0} \in H_{0}} h_{0} D$ by the Takagi Decomposition for complex symmetric matrices (see [28, Cor. 4.4.4], 33. Theorem 2], cf. also [34, Example 3.5]). It can be established that

$$
H=\left\{h=U(\cdot) A U^{T}: U \in \mathbb{U}_{n}, A=\operatorname{diag}\left(a_{1}, \ldots, a_{n}\right),\left|a_{i}\right|=1\right\} .
$$

It follows that condition (i) in Theorem 3.16 is fulfilled. Consequently, part (ii) of this theorem holds. For instance, for the operator $L: V \rightarrow V$ given by $L X=X^{T}$ one has $W=\{X \in V: L X=X\}$ and $L$ is $G$-doubly stochastic, since $L X \prec_{G} X$ for $X \in V$ by $s\left(X^{T}\right)=s(X)$ [29, p. 154].

On the other hand, the orthoprojector from $V$ onto $W$ is given by

$$
P X=\frac{X+X^{T}}{2} \quad \text { for } \quad X \in \mathbb{M}_{n}(\mathbb{C}) .
$$

By virtue of Theorems 3.16, 3.1 and 3.12 via the Takagi Decomposition one obtains

$$
s\left(\frac{X+X^{T}}{2}\right) \prec_{w} s(X) \quad \text { for } \quad X \in \mathbb{M}_{n}(\mathbb{C}) \text {. }
$$

4. Morphisms of E-systems. In this section we study properties and applications of morphisms of E-systems.

Unless otherwise stated, $V$ and $W$ are finite-dimensional real inner product spaces, and $G$ and $H$ are closed subgroups of the orthogonal groups $O(V)$ and $O(W)$, respectively.

4.1. Motivation. In 35] Lewis introduced the notion of isomorphic ND systems.

Definition 4.1 (Isomorphic ND systems). Two ND systems $\left(V, G,(\cdot)_{\downarrow}\right)$ and $\left(W, H,(\cdot)_{\downarrow}\right)$ are said to be isomorphic if there exist an inner product space isomorphism $K: V \rightarrow W$ and a group isomorphism $\varphi: G \rightarrow H$ such that $(K x)_{\downarrow}=K x_{\downarrow}$ and $K g x=\varphi(g) K x$ for $x \in V$ and $g \in G$ [35, p. 931].

In order to motivate our next definition, we give an example.

ExAmple 4.2. Let $(V, G, D)$ be the E-system defined in Example 2.18 It is known that

$$
s(A \circ X) \prec_{w} s(A) \circ s(X) \quad \text { for } A, X \in \mathbb{M}_{n}(\mathbb{C}),
$$


where $\circ$ denotes the Hadamard (entrywise) product of matrices in $\mathbb{M}_{n}(\mathbb{C})$ and of vectors in $\mathbb{R}^{n}$ (see [32, p. 168]).

Assume $A$ is a diagonal matrix with decreasingly ordered positive diagonal entries. Define the linear operator $K_{A}: \mathbb{M}_{n}(\mathbb{C}) \rightarrow \mathbb{M}_{n}(\mathbb{C})$ by

$$
K_{A} X=A \circ X \quad \text { for } X \in \mathbb{M}_{n}(\mathbb{C}) .
$$

Let $(W, H, E)=(V, G, D)$. Then $K_{A} D \subset E$ and inequality (11) leads to

$$
\left(K_{A} x\right)_{\downarrow} \prec_{H} K_{A} x_{\downarrow} \quad \text { for } x \in V .
$$

Likewise, the following result holds for the conventional product:

$$
s(A X) \prec_{w} s(A) \circ s(X) \text { for } A, X \in \mathbb{M}_{n}(\mathbb{C})
$$

(see [32, p. 168]). Taking

$$
K_{A} X=A X \quad \text { for } X \in \mathbb{M}_{n}(\mathbb{C})
$$

with diagonal matrix $A$ as above, we deduce from $\sqrt{13}$ that 12 is satisfied.

\subsection{Morphisms and simple morphisms}

Definition 4.3 (Morphism). Given two E-systems $\mathcal{E}=(V, G, D)$ and $\mathcal{F}=(W, H, E)$, a linear operator $K$ is said to be an $\mathcal{E}, \mathcal{F}$-morphism if

$$
K D \subset E \quad \text { and } \quad(K x)_{\downarrow} \prec_{H} K x_{\downarrow} \quad \text { for } x \in V .
$$

The set of all morphisms of E-systems $\mathcal{E}$ and $\mathcal{F}$ is denoted by $\operatorname{Mor}(\mathcal{E}, \mathcal{F})$.

The second part of condition (14) can be restated in the following equivalent form:

$$
y \prec_{G} x \quad \text { implies } K y \prec_{H} K x \quad \text { for } x \in D \text { and } y \in V \text {. }
$$

An important class of $\mathcal{E}, \mathcal{F}$-morphisms are simple morphisms.

Definition 4.4 (Simple morphism). Given two E-systems $\mathcal{E}=(V, G, D)$ and $\mathcal{F}=$ $(W, H, E)$, a linear operator $K: V \rightarrow W$ is said to be a simple morphism, if

$$
K D \subset E \quad \text { and } \quad K x \equiv_{H} K x_{\downarrow} \quad \text { for } x \in V
$$

or equivalently,

$$
K D \subset E \quad \text { and } \quad K g x \equiv_{H} K x \quad \text { for } x \in D \text { and } g \in G .
$$

The set of all simple morphisms of E-systems $\mathcal{E}$ and $\mathcal{F}$ is denoted by $\operatorname{SMor}(\mathcal{E}, \mathcal{F})$.

The set $\operatorname{Mor}(\mathcal{E}, \mathcal{F})$ of $\mathcal{E}, \mathcal{F}$-morphisms is a closed convex cone. The set Mor $\mathcal{E}=$ $\operatorname{Mor}(\mathcal{E}, \mathcal{E})$ is a selfadjoint semigroup.

Hereafter $K^{*}: W \rightarrow V$ is the dual operator (adjoint) of $K$ (see 10 ).

Theorem 4.5 ([55, Theorem 2.1]). Assume $\mathcal{E}=(V, G, D)$ and $\mathcal{F}=(W, H, E)$ are E-systems, and $K: V \rightarrow W$ is a linear operator. The following two conditions are equivalent:

(i) $K D \subset E$, and $K g x \prec_{H} K x$ for $x \in D$ and $g \in G$.

(ii) $K^{*} E \subset D$, and $K^{*} h z \prec_{G} K^{*} z$ for $z \in E$ and $h \in H$. 
Theorem 4.5 asserts that $K$ is a morphism if and only if $K^{*}$ is so. Furthermore,

$$
K \in \operatorname{Mor}(\mathcal{E}, \mathcal{F}) \quad \text { implies } \quad K^{*} K \in \operatorname{Mor}(\mathcal{E}) \quad \text { and } \quad K K^{*} \in \operatorname{Mor}(\mathcal{F}) .
$$

The next result suggests that by employing some easily checkable classes of morphisms $K^{*}$, one can get other morphisms $K$.

Theorem 4.6 ([55, Theorem 2.2]). Assume $\mathcal{E}=(V, G, D)$ and $\mathcal{F}=(W, H, E)$ are E-systems, and $K: V \rightarrow W$ is a linear operator. Suppose that $\mathcal{F}_{0}=\left(W_{0}, H_{0}, E_{0}\right)$ is a subsystem of $\mathcal{F}$ such that $K V \subset W_{0}$.

If $K^{*} \in \operatorname{Mor}\left(\mathcal{F}_{0}, \mathcal{E}\right)$, i.e., $K^{*} E_{0} \subset D$ and

$$
K^{*} h_{0} z \prec_{G} K^{*} z \quad \text { for } z \in E_{0} \text { and } h_{0} \in H_{0},
$$

then $K \in \operatorname{Mor}\left(\mathcal{E}, \mathcal{F}_{0}\right)$, i.e., $K D \subset E_{0}$ and

$$
K g x \prec_{H_{0}} K x \quad \text { for } x \in D \text { and } g \in G \text {. }
$$

In particular, if $K^{*} \in \operatorname{SMor}\left(\mathcal{F}_{0}, \mathcal{E}\right)$, i.e., $K^{*} E_{0} \subset D$ and

$$
\text { for } z \in E_{0} \text { and } h_{0} \in H_{0} \text { there exists } g \in G \text { such that } K^{*} h_{0} z=g K^{*} z \text {, }
$$

then $K \in \operatorname{Mor}\left(\mathcal{E}, \mathcal{F}_{0}\right)$.

For instance, if $\mathcal{E}=\mathcal{F}$ and the restriction of $K^{*}$ to $W_{0}$ is the identity, then 16 holds.

4.3. Homomorphisms. Throughout this subsection, $W_{0}$ is a finite-dimensional real inner product space, $H_{0}$ is a closed subgroup of the orthogonal group $O\left(W_{0}\right)$, and $E_{0}$ is a closed convex cone included in $W_{0}$. We define $\|w\|=\langle w, w\rangle^{1 / 2}$ for $w \in W_{0}$.

Our aim is to provide some sufficient conditions for triple $\mathcal{F}_{0}=\left(W_{0}, H_{0}, E_{0}\right)$ to be an E-system. We begin with axiom (A1).

THEOREM 4.7 ([55, Theorem 3.1]). The following three statements are equivalent:

(i) Axiom (A1) is satisfied for $\mathcal{F}_{0}=\left(W_{0}, H_{0}, E_{0}\right)$.

(ii) For any $w \in W_{0}$ there exists $u \in E_{0}$ such that $w \prec_{H_{0}} u$ and $\|w\|=\|u\|$.

(iii) There exist a triple $\mathcal{E}=(V, G, D)$ (not necessarily an E-system) and a linear operator $K: V \rightarrow W_{0}$ such that the following conditions are satisfied:

$$
\begin{gathered}
W_{0}=K V, \quad E_{0}=K D \text { and } H_{0} \text { is a closed subgroup of } O\left(W_{0}\right) . \\
\text { For } z \in V \text { and } x \in D, z \prec_{G} x \text { implies } K z \prec_{H_{0}} K x . \\
\text { For } w \in W_{0} \text { there exist } z \in V \text { and } x \in D \text { such that } \\
z \prec_{G} x, w=K z \text { and }\|w\|=\|K x\| .
\end{gathered}
$$

In the rest of this subsection, we investigate systems $\mathcal{F}_{0}=\left(W_{0}, H_{0}, E_{0}\right)$ of form (18), where $K: V \rightarrow W_{0}$ is a linear operator and $\mathcal{E}=(V, G, D)$ is a triple (not necessarily an E-system). In light of Theorem 4.7, we must concentrate on operators satisfying (19)-(20).

According to (14) and (15), we say that $K$ is an $\mathcal{E}, \mathcal{F}_{0}$-morphism if $(19)$ is met. (Here we do not assume that $\mathcal{E}$ and $\mathcal{F}_{0}$ are E-systems.)

Definition 4.8 (Radial morphism). An $\mathcal{E}, \mathcal{F}_{0}$-morphism $K$ is said to be $\mathcal{E}, \mathcal{F}_{0}$-radial morphism if 20 holds. 
Note that each simple morphism is a radial morphism.

Now we focus on axiom (A2) for the triple $\mathcal{F}_{0}=\left(W_{0}, H_{0}, E_{0}\right)$.

TheOREM 4.9 ([55, Theorem 3.3]). Let $\mathcal{E}=(V, G, D)$ be an E-system and let $K$ be an $\mathcal{E}, \mathcal{F}_{0}$-radial morphism.

The following two conditions are equivalent:

(i) Axiom (A2) is satisfied for $\mathcal{F}_{0}=\left(W_{0}, H_{0}, E_{0}\right)$.

(ii) The operator $K^{*}: W_{0} \rightarrow V$ is an $\mathcal{F}_{0}, E$-morphism, i.e., $K^{*} E_{0} \subset D$ and

$$
K^{*} h_{0} u \prec_{G} K^{*} u \quad \text { for } h_{0} \in H_{0} \text { and } u \in E_{0} .
$$

For triples $\mathcal{F}_{0}=\left(W_{0}, H_{0}, E_{0}\right)$ and $\mathcal{F}=(W, H, E)$, we write $\mathcal{F}_{0} \subset \mathcal{F}$ if

$$
W_{0} \subset W, \quad E_{0} \subset E \text { and }\left.\quad H_{0} \subset\left\{h \in H: h W_{0} \subset W_{0}\right\}\right|_{W_{0}} .
$$

Corollary 4.10 ([55, Corollary 3.4]). Let $\mathcal{E}=(V, G, D)$ be an E-system and let $K$ be an $\mathcal{E}, \mathcal{F}_{0}$-radial morphism.

If there exists an E-system $\mathcal{F}=(W, H, E)$ such that $\mathcal{F}_{0} \subset \mathcal{F}$, then $\mathcal{F}_{0}=\left(W_{0}, H_{0}, E_{0}\right)$ is an E-system.

Definition 4.11 (Homomorphism). An $\mathcal{E}, \mathcal{F}_{0}$-morphism $K$ is called an $\mathcal{E}, \mathcal{F}_{0}$-homomorphism if for $u \in E_{0}$ and $w \in W_{0}, w \equiv_{H_{0}} u$ implies that there exist $x \in D$ and $z \in V$ such that $z \prec_{G} x, u=K x$ and $w=K z$.

Theorem 4.12 provides further sufficient conditions for $\mathcal{F}_{0}=\left(W_{0}, H_{0}, E_{0}\right)$ to be an E-system (cf. [49, Theorems 3.4 and 3.9]).

TheOREM 4.12 ([55, Theorem 3.5]). Let $\mathcal{E}=(V, G, D)$ be an E-system and let $K$ be an $\mathcal{E}, \mathcal{F}_{0}$-radial homomorphism.

If $K^{*} K D \subset D$, then $\mathcal{F}_{0}=\left(W_{0}, H_{0}, E_{0}\right)$ is an E-system.

Now we consider partial isometries.

Definition 4.13 (Partial isometry). A linear operator $K: V \rightarrow W$ is said to be a partial isometry if $K K^{*} K=K$.

In the event that $K$ is a partial isometry, the operator $K K^{*}: W \rightarrow W$ is the orthoprojector from $W$ onto the subspace $K K^{*} W=K V=W_{0}$.

THEOREM 4.14 ([55, Theorem 3.6]). Let $K: V \rightarrow W$ be a partial isometry with finitedimensional real inner product spaces $V$ and $W$, and let $D \subset V$ be a closed convex cone. Let $\mathcal{F}_{0}=\left(W_{0}, H_{0}, E_{0}\right)$, where $W_{0}=K V, E_{0}=K D$ and $H_{0}$ is a closed subgroup of $O\left(W_{0}\right)$. Suppose that $\mathcal{F}=(W, H, E)$ is an E-system such that $\mathcal{F}_{0} \subset \mathcal{F}$ and $K K^{*} E=E_{0}$.

Then the following statements are equivalent:

(i) $\mathcal{F}_{0}=\left(W_{0}, H_{0}, E_{0}\right)$ is an E-system.

(ii) $K K^{*} w \prec_{H_{0}} K K^{*} w_{\downarrow}$ for $w \in W$, where $(\cdot)_{\downarrow}$ stands for the normal map of $\mathcal{F}$.

(iii) $W_{0} \subset \bigcup_{h \in H} h E_{0}$, and, in addition, the operator $K K^{*}$ is an $\mathcal{F}, \mathcal{F}_{0}$-radial homomorphism. 
4.4. Applications to matrices. We illustrate Theorem 4.6 for the matrix E-system $\mathcal{E}=(V, G, D)$ defined in Example 2.18 and their subsystems. Let $\mathcal{F}=(W, H, E)$ with $W=V, H=G$ and $E=D$.

Some subsystems $\mathcal{F}_{0}=\left(W_{0}, H_{0}, E_{0}\right)$ of $\mathcal{F}$ are collected below (see [15, 16, 35, 70]).

(I) $W_{0}=\mathbb{M}_{n}(\mathbb{R})=$ the space of $n \times n$ real matrices,

$H_{0}=$ the group of orthogonal equivalences $U_{1}(\cdot) U_{2}$ with $U_{1}, U_{2} \in \mathbb{O}_{n}$,

$E_{0}=\left\{\operatorname{diag}\left(s_{1}, \ldots, s_{n}\right): s_{1} \geq \ldots \geq s_{n} \geq 0\right\}$.

(II) $W_{0}=\mathbb{S}_{n}(\mathbb{C})=$ the space of $n \times n$ complex symmetric matrices,

$H_{0}=$ the group of unitary congruences $U(\cdot) U^{T}$ with $U \in \mathbb{U}_{n}$,

$E_{0}=\left\{\operatorname{diag}\left(s_{1}, \ldots, s_{n}\right): s_{1} \geq \ldots \geq s_{n} \geq 0\right\}$.

(III) $W_{0}=$ the space of $n \times n$ matrices of the form $\left(\begin{array}{cc}X & 0 \\ 0 & 0\end{array}\right)$ with $k \times k$ matrix $X \in \mathbb{M}_{k}(\mathbb{C})$ and $1 \leq k \leq n$,

$H_{0}=$ the group of unitary similarities $U_{1}(\cdot) U_{2}$ with $U_{1}$ and $U_{2}$ being the matrix of the form $\left(\begin{array}{cc}U & 0 \\ 0 & I_{n-k}\end{array}\right)$ for some $k \times k$ unitary matrix $U$,

$E_{0}=\left\{\operatorname{diag}\left(s_{1}, \ldots, s_{k}, 0, \ldots, 0\right): s_{1} \geq \ldots \geq s_{k} \geq 0\right\}$.

(IV) $W_{0}=\mathbb{D}_{n}(\mathbb{C})=$ the space of $n \times n$ complex diagonal matrices,

$H_{0}=$ the group of equivalences $U_{1}(\cdot) U_{2}$ with $U_{1}, U_{2} \in \mathbb{G P}_{n}(\mathbb{C})$,

$E_{0}=\left\{\operatorname{diag}\left(s_{1}, \ldots, s_{n}\right): s_{1} \geq \ldots \geq s_{n} \geq 0\right\}$.

(V) $W_{0}=\mathbb{D}_{n}(\mathbb{R})=$ the space of $n \times n$ real diagonal matrices,

$H_{0}=$ the group of equivalences $U_{1}(\cdot) U_{2}$ with $U_{1}, U_{2} \in \mathbb{G P}_{n}(\mathbb{R})$,

$E_{0}=\left\{\operatorname{diag}\left(s_{1}, \ldots, s_{n}\right): s_{1} \geq \ldots \geq s_{n} \geq 0\right\}$.

Corollary 4.15 ([55, Corollary 4.1]). For any of the above subsystems $\mathcal{F}_{0}$, let $K$ : $\mathbb{M}_{n}(\mathbb{C}) \rightarrow \mathbb{M}_{n}(\mathbb{C})$ be a linear operator such that $K \mathbb{M}_{n}(\mathbb{C}) \subset W_{0}$.

(i) If the restriction $\left.K^{*}\right|_{W_{0}}$ is a simple morphism of $\mathcal{F}_{0}$ and $\mathcal{E}$, i.e., if (17) is satisfied and $K^{*} E_{0} \subset D$, then $K$ is a morphism of $\mathcal{E}$ and $\mathcal{F}_{0}$, i.e., $K D \subset E_{0}$ and

$$
s(K x) \prec_{w} s\left(K x_{\downarrow}\right) \quad \text { for } x \in \mathbb{M}_{n}(\mathbb{C}),
$$

where $x_{\downarrow}=\operatorname{diag} s(x)$.

(ii) If the restriction $\left.K^{*}\right|_{W_{0}}$ is the identity on $W_{0}$, then inequality (21) holds.

(iii) If $K$ is symmetric $\left(K^{*}=K\right)$ and $D \subset W_{0}$, and if the restriction $\left.K\right|_{W_{0}}$ is the identity, then inequality (21) holds in the form

$$
s(K x) \prec_{w} s(x) \quad \text { for } x \in \mathbb{M}_{n}(\mathbb{C}) .
$$

For the subsystem defined in (IV), 22 generalizes the classical Ky Fan's inequality 4 .

The next result is a consequence of Theorem 4.14

Corollary 4.16 ([55, Corollary 4.2]). Let $K: \mathbb{M}_{n}(\mathbb{C}) \rightarrow \mathbb{M}_{n}(\mathbb{C})$ be a partial isometry. Let $W_{0}=K V, E_{0}=K D$ and $H_{0}$ be a closed subgroup of $O\left(W_{0}\right)$. Suppose that $K K^{*} D=E_{0}$. Then

$$
\mathcal{F}_{0}=\left(W_{0}, H_{0}, E_{0}\right) \text { is an E-system iff } \quad K K^{*} w \prec_{H_{0}} K K^{*} w_{\downarrow} \text { for } w \in \mathbb{M}_{n}(\mathbb{C}),
$$

where $w_{\downarrow}=\operatorname{diag} s(w)$. 


\section{Chebyshev functional and its applications}

\subsection{Motivation}

Definition 5.1 (Synchronous functions). Two functions $f, g:[a, b] \rightarrow \mathbb{R}$ are said to be synchronous if

$$
[f(t)-f(u)][g(t)-g(u)] \geq 0 \quad \text { for } t, u \in[a, b] .
$$

The celebrated Chebyshev integral inequality says that if real functions $f, g \in L_{[a, b]}^{2}$ are synchronous then

$$
(b-a) \int_{a}^{b} f(t) g(t) d t-\int_{a}^{b} f(t) d t \cdot \int_{a}^{b} g(t) d t \geq 0 .
$$

Note that the coefficient $b-a$ in 23 satisfies $\int_{a}^{b} v^{2}(t) d t=b-a$ with $v(t)=1$, $t \in[a, b]$.

It is obvious that each two nonincreasing functions $f, g:[a, b] \rightarrow \mathbb{R}$ are synchronous. Thus Chebyshev inequality (23) is true for two nonincreasing functions.

There are many inequalities similar to (23), e.g., Andersson's result [1] asserts that if $f, g:[0,1] \rightarrow \mathbb{R}$ and $f(0)=g(0)=0$ and both $f$ and $g$ are increasing and convex then

$$
\frac{3}{4} \int_{0}^{1} f(t) g(t) d t-\int_{0}^{1} f(t) d t \cdot \int_{0}^{1} g(t) d t \geq 0 .
$$

Fink 22] proved that

$$
\frac{\left(\int_{0}^{1} t d \sigma(t)\right)^{2}}{\int_{0}^{1} t^{2} d \sigma(t)} \int_{0}^{1} f(t) g(t) d \sigma(t)-\int_{0}^{1} f(t) d \sigma(t) \cdot \int_{0}^{1} g(t) d \sigma(t) \geq 0
$$

for functions

$$
f, g \in\left\{h \in C_{[0,1]}^{1}: h(0)=0 \text { and } h(t) / t \text { is increasing on }(0,1]\right\}
$$

and measures $\sigma$ such that

$$
\int_{0}^{s} t d \sigma(t) \geq 0 \quad \text { and } \quad \int_{s}^{1} t d \sigma(t) \geq 0 \quad \text { for } s \in[0,1], \quad \text { and } \quad \int_{0}^{1} t d \sigma(t)>0 .
$$

A discrete counterpart of (24) is the following Toader's weighted inequality [73]:

$$
\frac{\left(\sum_{i=1}^{n} i v_{i}\right)^{2}}{\sum_{i=1}^{n} i^{2} v_{i}} \sum_{i=1}^{n} x_{i} y_{i} v_{i}-\sum_{i=1}^{n} x_{i} v_{i} \cdot \sum_{i=1}^{n} y_{i} v_{i} \geq 0
$$

provided that $v_{i}>0, i=1,2, \ldots, n$, and $x$ and $y$ are star-shaped, i.e., the sequences $i \rightarrow x_{i} / i$ and $i \rightarrow y_{i} / i$ are nondecreasing (see [51, p. 239]).

Definition 5.2 (Synchronous $n$-tuples). Two $n$-tuples $x=\left(x_{1}, \ldots, x_{n}\right) \in \mathbb{R}^{n}$ and $y=\left(y_{1}, \ldots, y_{n}\right) \in \mathbb{R}^{n}$ are said to be synchronous if

$$
\left(x_{i}-x_{j}\right)\left(y_{i}-y_{j}\right) \geq 0 \quad \text { for } i, j \in\{1,2, \ldots, n\} .
$$

A discrete version of 23 is as follows. If $x, y \in \mathbb{R}^{n}$ are synchronous then

$$
n \sum_{i=1}^{n} x_{i} y_{i}-\sum_{i=1}^{n} x_{i} \cdot \sum_{i=1}^{n} y_{i} \geq 0 .
$$

This is the Chebyshev sum inequality. 
The coefficient $n$ in 26 satisfies $\sum_{i=1}^{n} v_{i}^{2}=n$, where $v_{i}=1$ for $i=1,2, \ldots, n$, and the unital vector $v=(1, \ldots, 1) \in \mathbb{R}^{n}$ is a common fixed point for all operators in the group $\mathbb{P}_{n}$ of $n \times n$-permutation matrices, i.e., $v \in\left\{a \in \mathbb{R}^{n}: p a=a\right.$ for $\left.p \in \mathbb{P}_{n}\right\}$.

In Subsections 5.2 5.3 we show methods for establishing inequalities similar to (23) - 26) with the help of the Chebyshev functional, Eaton triples and the notion of similarly separable vectors.

Throughout this section, unless otherwise stated, $V$ is a finite-dimensional real linear space with an inner product $\langle\cdot, \cdot\rangle$, and $O(V)$ denotes the orthogonal group acting on $V$.

5.2. Chebyshev functional and E-systems. Here we present Chebyshev type inequalities in the framework of E-systems.

Definition 5.3 (Chebyshev functional). The Chebyshev functional is defined by

$$
T_{v}(x, y)=\langle v, v\rangle\langle x, y\rangle-\langle x, v\rangle\langle y, v\rangle \quad \text { for } x, y \in V,
$$

where $0 \neq v \in V$ [50, p. 535].

Definition 5.4 ( $G$-synchronous vectors). Given an Eaton triple $(V, G, D)$, two vectors $x, y \in V$ are said to be $G$-synchronous if there exists $g \in G$ such that $x, y \in g D$.

We define

$$
M_{G}(V)=\{a \in V: g a=a \text { for } g \in G\} .
$$

Theorem 5.5 ([50, Theorem 3.1]). Let $(V, G, D)$ be an Eaton triple with one-dimensional subspace $M_{G}(V)=\operatorname{span} v$, where $0 \neq v \in V$.

If $x, y \in V$ are $G$-synchronous then the following Chebyshev type inequality holds:

$$
\langle v, v\rangle\langle x, y\rangle-\langle x, v\rangle\langle y, v\rangle \geq 0 .
$$

In the forthcoming examples we give some interpretations of inequality 28 .

ExAmPle 5.6. Let $(V, G, D)=\left(\mathbb{R}^{n}, \mathbb{P}_{n}, D\right)$, where $D=\left\{a=\left(a_{1}, \ldots, a_{n}\right) \in \mathbb{R}^{n}: a_{1} \geq\right.$ $\left.a_{2} \geq \ldots \geq a_{n}\right\}$. Then $M_{G}(V)=\operatorname{span} v$ for $v=(1, \ldots, 1) \in \mathbb{R}^{n}$.

Let $\left(\left(x_{1}, y_{1}\right), \ldots,\left(x_{n}, y_{n}\right)\right)$ be a sample of size $n$ from distribution of a two-dimensional random vector. If $x=\left(x_{1}, \ldots, x_{n}\right)$ and $y=\left(y_{1}, \ldots, y_{n}\right)$ are synchronous then $x$ and $y$ are positively correlated, i.e.,

$$
S_{x, y}=\frac{1}{n} \sum_{i=1}^{n} x_{i} y_{i}-\frac{1}{n} \sum_{i=1}^{n} x_{i} \cdot \frac{1}{n} \sum_{i=1}^{n} y_{i} \geq 0
$$

(see $26-28)$.

ExAmple 5.7 ([50, pp. 540-541]). Let $(V, G, D)$ be the Eaton triple defined in Example 2.17. Then $M_{G}(V)=\operatorname{span} I_{n}$, where $I_{n}$ denotes the $n$-by- $n$ identity matrix. The $G$-synchronicity of Hermitian matrices $X$ and $Y$ means that $X$ and $Y$ are simultaneously diagonalizable. Additionally, $X_{\downarrow}=\operatorname{diag} \lambda(X)$, where $\lambda(X)=\left(\lambda_{1}(X), \ldots, \lambda_{n}(X)\right)$ is the vector of the eigenvalues of a Hermitian matrix $X$ with $\lambda_{1}(X) \geq \ldots \geq \lambda_{n}(X)$.

In light of Theorem 5.5 the following Chebyshev type inequality holds:

$$
n \sum_{i=1}^{n} \lambda_{i}(X) \lambda_{i}(Y) \geq \operatorname{tr} X \operatorname{tr} Y \quad \text { for } X, Y \in \mathbb{H}_{n} .
$$


5.3. Generalized Chebyshev functional. Motivated by Theorem 5.5, we study a generalization of Chebyshev functional (27).

Definition 5.8 (Generalized Chebyshev functional). Assume $(V,\langle\cdot, \cdot\rangle)$ is an inner product space. The generalized Chebyshev functional is defined by

$$
T_{v, w}(x, y)=\langle v, w\rangle\langle x, y\rangle-\langle x, v\rangle\langle y, w\rangle \quad \text { for } x, y \in V,
$$

where $0 \neq v, w \in V$.

We are interested in sufficient and necessary conditions for the functional $[29$ to be nonnegative. As will be seen below, the key property is the separability of some vectors. Separable vectors are natural generalizations of monotone, monotone in mean, star-shaped and convex sequences, etc.

Definition 5.9 (Separable vector). Let $(V,\langle\cdot, \cdot\rangle)$ be an inner product space and $e=$ $\left(e_{1}, \ldots, e_{n}\right) \in V^{n}, v \in V$ and $\mu \in \mathbb{R}$. Let $J_{1}$ and $J_{2}$ be index sets with $J_{1} \cup J_{2}=$ $\{1,2, \ldots, n\}$.

A vector $z \in V$ is said to be $\mu, v$-separable on $J_{1}$ and $J_{2}$ with respect to $e$, if

$$
\left\langle z-\mu v, e_{i}\right\rangle \geq 0 \quad \text { for } i \in J_{1}, \quad \text { and } \quad\left\langle z-\mu v, e_{j}\right\rangle \leq 0 \text { for } j \in J_{2}
$$

(see [51, p. 235]).

Note that if $\left\langle v, e_{i}\right\rangle>0$ for $i=1,2, \ldots, n$ then the $\mu, v$-separability of vector $z$ is equivalent to

$$
\frac{\left\langle z, e_{i}\right\rangle}{\left\langle v, e_{i}\right\rangle} \geq \mu \geq \frac{\left\langle z, e_{j}\right\rangle}{\left\langle v, e_{j}\right\rangle} \quad \text { for } i \in J_{1} \text { and } j \in J_{2} .
$$

The three examples below include interpretations of the notion of separability in $V=\mathbb{R}^{n}$ with the standard inner product

$$
\langle a, b\rangle=\sum_{k=1}^{n} a_{k} b_{k} \quad \text { for } a=\left(a_{1}, a_{2}, \ldots, a_{n}\right) \text { and } b=\left(b_{1}, b_{2}, \ldots, b_{n}\right) .
$$

EXAMPLE 5.10. If $z=\left(z_{1}, \ldots, z_{n}\right) \in \mathbb{R}^{n}, v=(1,1, \ldots, 1) \in \mathbb{R}^{n}$ and $e=\left(e_{1}, \ldots, e_{n}\right)$ is the standard basis of $\mathbb{R}^{n}$, i.e., $e_{k}=(\underbrace{0, \ldots, 0}_{k-1 \text { times }}, 1,0, \ldots, 0) \in \mathbb{R}^{n}$ for $k=1,2, \ldots, n$, then condition means that

$$
z_{i} \geq \mu \geq z_{j} \quad \text { for } i \in J_{1} \text { and } j \in J_{2} .
$$

EXAMPLE 5.11 ([59, p. 937]). Remind that a vector $z=\left(z_{1}, z_{2}, \ldots, z_{n}\right) \in \mathbb{R}^{n}$ is said to be star-shaped if

$$
\frac{z_{1}}{1} \leq \frac{z_{2}}{2} \leq \ldots \leq \frac{z_{n}}{n}
$$

Choose $v=(1,2, \ldots, n)$. Then 33 can be rewritten as

$$
\frac{\left\langle z, e_{1}\right\rangle}{\left\langle v, e_{1}\right\rangle} \leq \frac{\left\langle z, e_{2}\right\rangle}{\left\langle v, e_{2}\right\rangle} \leq \ldots \leq \frac{\left\langle z, e_{n}\right\rangle}{\left\langle v, e_{n}\right\rangle}
$$

For arbitrary $m \in\{0,1, \ldots, n\}$, take $\mu$ to be any number between $\frac{\left\langle z, e_{m}\right\rangle}{\left\langle v, e_{m}\right\rangle}$ and $\frac{\left\langle z, e_{m+1}\right\rangle}{\left\langle v, e_{m}+1\right.}$ (with the convention that $\frac{\left\langle z, e_{0}\right\rangle}{\left\langle v, e_{0}\right\rangle}=-\infty$ and $\frac{\left\langle z, e_{n+1}\right\rangle}{\left\langle v, e_{n+1}\right\rangle}=\infty$ ). Then 31] is fulfilled for 
$J_{1}=\{m+1, m+2, \ldots, n\}$ and $J_{2}=\{1,2, \ldots, m\}$. In other words, $z$ is $\mu, v$-separable on $J_{1}$ and $J_{2}$ with respect to $e$ for each $m \in\{0,1, \ldots, n\}$.

EXAMPLE 5.12 ([59, p. 937]). We show that the majorization relation $x \prec y$ corresponds to the $\mu$-separability of the vector $y-x$ for $\mu=0$ and $J_{1}=\{1,2, \ldots, n\}$ and $J_{2}=\{n\}$.

Let $x=\left(x_{1}, x_{2}, \ldots, x_{n}\right) \in \mathbb{R}^{n}$ and $y=\left(y_{1}, y_{2}, \ldots, y_{n}\right) \in \mathbb{R}^{n}$ with $x_{1} \geq x_{2} \geq \ldots \geq x_{n}$ and $y_{1} \geq y_{2} \geq \ldots \geq y_{n}$. Suppose that $x$ is majorized by $y$. That is,

$$
\sum_{i=1}^{k}\left(y_{i}-x_{i}\right) \geq 0=\sum_{i=1}^{n}\left(y_{i}-x_{i}\right) \quad \text { for } k=1,2, \ldots, n .
$$

Put $v=(1, \ldots, 1) \in \mathbb{R}^{n}$ and $e_{k}=(\underbrace{1, \ldots, 1}_{k \text { times }}, 0, \ldots, 0) \in \mathbb{R}^{n}$ for $k=1,2, \ldots, n$. Then (34) reads

$$
\left\langle y-x, e_{k}\right\rangle \geq 0=\left\langle y-x, e_{n}\right\rangle \quad \text { for } k=1,2, \ldots, n .
$$

With $\mu=0$ and $J_{1}$ and $J_{2}$ defined above, one has

$$
\left\langle y-x-\mu v, e_{i}\right\rangle \geq 0=\left\langle y-x-\mu v, e_{j}\right\rangle \quad \text { for } i \in J_{1} \text { and } j \in J_{2} .
$$

Therefore the difference $z=y-x$ is $0, v$-separable on $J_{1}$ and $J_{2}$ w.r.t. $e$ (see $(30)$ ).

We define

$S_{e}\left(v ; J_{1}, J_{2}\right)=\left\{z \in V: z\right.$ is $v$-separable on $J_{1}$ and $J_{2}$ w.r.t. $e$ for some $\left.\mu \in \mathbb{R}\right\}$.

The role of the separability in determining the sign of the generalized Chebyshev functional 29 is shown in the following result.

TheOREM $5.13([48$, Theorem 3.5]). Let $(V,\langle\cdot, \cdot\rangle)$ be a finite-dimensional real inner product space with two dual bases $e=\left(e_{1}, \ldots, e_{n}\right)$ and $d=\left(d_{1}, \ldots, d_{n}\right)$ in $V$, i.e., $\left\langle e_{i}, d_{j}\right\rangle=\delta_{i j}$ (Kronecker delta), $i, j \in J=\{1,2, \ldots, n\}$.

Let $y, w, v \in V$ with $\langle w, v\rangle>0$. Suppose that $J_{1}$ and $J_{2}$ are index sets with $J_{1} \cup J_{2}=J$.

Then the following two statements are equivalent:

(i) The generalized Chebyshev inequality

$$
\langle w, v\rangle\langle z, y\rangle-\langle z, w\rangle\langle y, v\rangle \geq 0
$$

holds for all $z \in S_{e}\left(v ; J_{1}, J_{2}\right)$.

(ii) The vector $y$ is $\eta$, $w$-separable on $J_{1}$ and $J_{2}$ w.r.t. $d$, where $\eta=\langle y, v\rangle /\langle w, v\rangle$.

The next definition is inspired by Theorem 5.13 .

Definition 5.14 (Similar separability). Let $e=\left(e_{1}, \ldots, e_{n}\right)$ and $d=\left(d_{1}, \ldots, d_{n}\right)$ be two sequences of vectors in $V$. Assume $v, w \in V$ and $\mu, \eta \in \mathbb{R}$.

Two vectors $a, b \in V$ are said to be similarly separable w.r.t. $(\mu, v, e ; \eta, w, d)$ if there exist index sets $J_{1}$ and $J_{2}$ with $J_{1} \cup J_{2}=\{1,2, \ldots, n\}$ such that

(i) $a$ is $\mu, v$-separable on $J_{1}$ and $J_{2}$ w.r.t. $e$, and

(ii) $b$ is $\eta, w$-separable on $J_{1}$ and $J_{2}$ w.r.t. $d$

(see [59, p. 937]). 
COROLlaRY 5.15 ([54, Lemma 2.1]). Let $(V,\langle\cdot, \cdot\rangle)$ be a finite-dimensional real inner product space with two dual bases $e=\left(e_{1}, \ldots, e_{n}\right)$ and $d=\left(d_{1}, \ldots, d_{n}\right)$ in $V$. Let $z, y, w, v \in V$ with $\langle w, v\rangle>0$. Define $\eta=\langle y, v\rangle /\langle w, v\rangle$.

If the vectors $z, y \in V$ are similarly separable w.r.t. $(\mu, v, e ; \eta, w, d)$ for some $\mu \in \mathbb{R}$, then the generalized Chebyshev inequality (35) holds.

It is interesting that the generalized Chebyshev inequality 35 contains CauchySchwarz inequality. Namely, if $y=z, w=v$ and $d=e$, then the similar separability of $z, y$ holds automatically for $\mu=\eta$. In this case (35) reduces to the $\mathrm{C}-\mathrm{S}$ inequality

$$
\|z\|^{2}\|v\|^{2} \geq\langle z, v\rangle^{2} \quad \text { for } z, v \in V \text {. }
$$

6. Applications of similar separability. In this section we apply Theorem 5.13 to give some generalizations of the classical Hardy-Littlewood-Pólya (H-L-P) Theorem for convex functions and of Schur-Ostrowski Theorem for differentiable Schur-convex functions. In addition, we deal with extended $G$-majorization.

6.1. Generalized $\mathbf{H}-\mathbf{L}-\mathbf{P}$ theorems. We begin with a result of $\mathrm{H}-\mathrm{L}-\mathrm{P}$ giving relationship between majorization and convexity.

TheOREM $6.1([27])$. Let $x, y \in \mathbb{R}^{n}$ with $x_{i}, y_{i} \in I$, where $I \subset \mathbb{R}$ is an interval.

The following two statements are equivalent:

(i) $y \prec x$.

(ii) The following inequality holds for all continuous convex functions $f: I \rightarrow \mathbb{R}$ :

$$
\sum_{k=1}^{n} f\left(y_{k}\right) \leq \sum_{k=1}^{n} f\left(x_{k}\right)
$$

Some extensions of Theorem 6.1 can be found in [11, 44].

For a given positive vector $\left(p_{1}, \ldots, p_{n}\right) \in \mathbb{R}_{+}^{n}$, we introduce inner product on $\mathbb{R}^{n}$ by

$$
\langle a, b\rangle=\sum_{k=1}^{n} a_{k} b_{k} p_{k} \quad \text { for } a=\left(a_{1}, \ldots, a_{n}\right), b=\left(b_{1}, \ldots, b_{n}\right) \in \mathbb{R}^{n} .
$$

TheOrem 6.2 ([54, Theorem 2.2]). Assume $f: I \rightarrow \mathbb{R}$ is a convex function on the open interval $I \subset \mathbb{R}$. Let $x=\left(x_{1}, \ldots, x_{n}\right), y=\left(y_{1}, \ldots, y_{n}\right)$ and $p=\left(p_{1}, \ldots, p_{n}\right)$, where $x_{i}, y_{i} \in I, p_{i}>0$ for $i \in J=\{1, \ldots, n\}$.

Let $\partial f: I \rightarrow \mathbb{R}$ be the subdifferential of $f$, and let $\varphi \in \partial f$. Define

$$
\Phi(z)=\left(\varphi\left(z_{1}\right), \ldots, \varphi\left(z_{n}\right)\right) \quad \text { for } z=\left(z_{1}, \ldots, z_{n}\right) \in I^{n} .
$$

Let $e$ and $d$ be dual bases for $\mathbb{R}^{n}$ with inner product given by $(36)$, and $w, v \in \mathbb{R}^{n}$ with $\langle w, v\rangle>0$. Let $\eta=\langle x-y, v\rangle /\langle w, v\rangle$.

Suppose that there exist index sets $J_{1}$ and $J_{2}$ with $J_{1} \cup J_{2}=J$ such that

(i) $y$ is $v$-separable on $J_{1}$ and $J_{2}$ w.r.t. $e$,

(ii) $x-y$ is $\eta$,w-separable on $J_{1}$ and $J_{2}$ w.r.t. $d$, and

(iii) $\Phi$ preserves $v$-separability on $J_{1}$ and $J_{2}$ w.r.t. e.

Under the above assumptions, the following assertions hold. 
(A) If $\langle x-y, v\rangle=0$, then

$$
\sum_{k=1}^{n} p_{k} f\left(y_{k}\right) \leq \sum_{k=1}^{n} p_{k} f\left(x_{k}\right)
$$

(B) If $\langle x-y, v\rangle \geq 0$ and $\langle\Phi(y), w\rangle \geq 0$, then (37) holds.

6.2. Generalization of Schur-Ostrowski Theorem. Characterizations of differentiable S-convex and $G$-increasing functions are provided below in Schur-Ostrowski's Theorem 6.3 and in Eaton-Perlman's Theorem 6.4 respectively.

THEOREM $6.3([66,62])$. Assume that $F$ is a symmetric real function having a differential on $\mathbb{R}^{n}$. Then a necessary and sufficient condition that $F$ be a Schur-convex function on $\mathbb{R}^{n}$ is

$$
\left(z_{i}-z_{j}\right)\left(\frac{\partial F}{\partial z_{i}}(z)-\frac{\partial F}{\partial z_{j}}(z)\right) \geq 0 \quad \text { for } z \in \mathbb{R}^{n} \text { and } i, j=1,2, \ldots, n .
$$

Statement (38) is called Schur-Ostrowski's condition (in short, $S-O$ condition).

TheOREm 6.4 ([18]). Let $G$ be a reflection group acting on $\mathbb{R}^{n}$. Assume that $F$ is a $G$ invariant real function possessing a differential on $\mathbb{R}^{n}$. Then a necessary and sufficient condition that $F$ be $G$-increasing on $\mathbb{R}^{n}$ is

$$
\langle z, r\rangle \cdot\langle\nabla F(z), r\rangle \geq 0 \quad \text { for } z \in \mathbb{R}^{n} \text { and } r \in \mathbb{R}^{n} \text { such that } S_{r} \in G,
$$

where $\nabla F(z)$ denotes the gradient of $F$ at $z$.

Let $(V,\langle\cdot, \cdot\rangle)$ be a real Hilbert space with norm $\|\cdot\|=\langle\cdot, \cdot\rangle^{1 / 2}$.

For a function $F: A \rightarrow \mathbb{R}$ with convex $A \subset V$, the symbol $\nabla_{h} F(z)$ stands for the directional derivative of $F$ in the direction $h \in V$ at the point $z$, and $\nabla F(z)$ stands for the gradient of $F$ at $z$.

Definition 6.5 (Generalized Schur-Ostrowski's condition). Given a convex set $A \subset V$ and $x, y \in A$ and $v \in V$, a differentiable function $F: A \rightarrow \mathbb{R}$ is said to satisfy generalized Schur-Ostrowski condition (GSOC) if for each $\mu \in \mathbb{R}$ and $z \in[x, y]$ there exists $\widetilde{\mu} \in \mathbb{R}$ such that

$$
\left\langle z-\mu v, e_{i}\right\rangle \cdot\left\langle\nabla F(z)-\tilde{\mu} v, e_{i}\right\rangle \geq 0 \quad \text { for } i=1,2, \ldots, n
$$

(see [59]).

Definition 6.6 (Function class $\mathcal{S}(A, x, y)$ satisfying GSOC). Given a convex set $A \subset V$ and $x, y \in A$ and $v, w \in V$, by $\mathcal{S}(A, x, y)$ we denote the class of all differentiable functions $F: A \rightarrow \mathbb{R}$ satisfying the generalized Schur-Ostrowski condition (GSOC) and such that the maps $[0,1] \ni t \rightarrow \nabla_{y-x} F(x+t(y-x))$ and $[0,1] \ni t \rightarrow \nabla_{w} F(x+t(y-x))$ are integrable on $[0,1]$ (see [59, p. 939]).

Members of the class $\mathcal{S}(A, x, y)$ are called generalized Schur-convex functions.

In the forthcoming results we generalize the sufficiency part of Theorems 6.3 and 6.4 . Theorem 6.7 ([59, Theorem 4]). Let $W$ be a finite-dimensional subspace of $V$ and $e=$ $\left(e_{1}, \ldots, e_{n}\right)$ and $d=\left(d_{1}, \ldots, d_{n}\right)$ be dual bases in $W$. Let $A \subset V$ be a convex set and let $x, y \in A$ and $w, v \in V$ with $\langle w, v\rangle>0$. 
Suppose that for some index sets $J_{1}$ and $J_{2}$ with $J_{1} \cup J_{2}=\{1,2, \ldots, n\}$ and for some $\mu_{1}, \mu_{2} \in \mathbb{R}$ and $\eta=\langle y-x, v\rangle /\langle w, v\rangle$,

$$
\begin{array}{rlll}
\left\langle x-\mu_{1} v, e_{i}\right\rangle>0 & \text { for } i \in J_{1} & \text { and } & \left\langle x-\mu_{1} v, e_{j}\right\rangle<0 \quad \text { for } j \in J_{2}, \\
\left\langle y-\mu_{2} v, e_{i}\right\rangle>0 & \text { for } i \in J_{1} & \text { and } & \left\langle y-\mu_{2} v, e_{j}\right\rangle<0 \quad \text { for } j \in J_{2}, \\
\left\langle y-x-\eta w, d_{i}\right\rangle \geq 0 & \text { for } i \in J_{1} & \text { and } & \left\langle y-x-\eta w, d_{j}\right\rangle \leq 0 \quad \text { for } j \in J_{2} .
\end{array}
$$

Let $F \in \mathcal{S}(A, x, y)$. Assume $y-x-\eta w \in W$ and $\nabla F(z)-\tilde{\mu} v \in W$ for $z \in[x, y]$. Under the above assumptions, the following three assertions hold.

(A) $F(y)-F(x) \geq \frac{\langle y-x, v\rangle}{\langle w, v\rangle} \int_{0}^{1}\langle\nabla F(x+t h), w\rangle d t$.

(B) If $\langle y-x, v\rangle=0$ then $F(x) \leq F(y)$.

(C) If $\langle y-x, v\rangle \geq 0$ and $\langle\nabla F(z), w\rangle \geq 0$ for $z \in[x, y]$, then $F(x) \leq F(y)$.

Theorem 6.7 can be simplified as follows.

Corollary 6.8 ([59, Corollaries 9-10]). The assertions (A), (B) and (C) of Theorem 6.7 are still true if the conditions (39), 40, and 41) are replaced by

$$
\begin{gathered}
\frac{\left\langle x, e_{1}\right\rangle}{\left\langle v, e_{1}\right\rangle}>\frac{\left\langle x, e_{2}\right\rangle}{\left\langle v, e_{2}\right\rangle}>\ldots>\frac{\left\langle x, e_{n}\right\rangle}{\left\langle v, e_{n}\right\rangle}, \\
\frac{\left\langle y, e_{1}\right\rangle}{\left\langle v, e_{1}\right\rangle}>\frac{\left\langle y, e_{2}\right\rangle}{\left\langle v, e_{2}\right\rangle}>\ldots>\frac{\left\langle y, e_{n}\right\rangle}{\left\langle v, e_{n}\right\rangle}, \\
\frac{\left\langle y-x, d_{1}\right\rangle}{\left\langle w, d_{1}\right\rangle} \geq \frac{\left\langle y-x, d_{2}\right\rangle}{\left\langle w, d_{2}\right\rangle} \geq \ldots \geq \frac{\left\langle y-x, d_{n}\right\rangle}{\left\langle w, d_{n}\right\rangle}
\end{gathered}
$$

(provided $v$ is e-positive and $w$ is d-positive).

An application of Theorem 6.7 to GIC orderings is included in the next result.

TheOrem 6.9 ([59, Theorem 13]). Let $\prec_{G}$ be a GIC ordering on $V$ induced by compact group $G \subset O(V)$ and closed convex cone $D \subset V$.

Let $V_{0}$ be a subspace of $V$ and $\left(e_{1}, \ldots, e_{n}\right)$ and $\left(s_{1}, \ldots, s_{n}\right)$ be dual bases in $V_{0}$ such that $C=$ cone $\left\{e_{1}, \ldots, e_{n_{1}}\right\}$ and $D=$ cone $\left\{s_{1}, \ldots, s_{n_{2}}\right\}$ are dual cones in $V_{0}$ with $n_{1} \leq$ $n \leq n_{2}$, where $s_{n+1}, \ldots, s_{n_{2}} \in V$.

Assume $w, v \in V$ with $\langle w, v\rangle>0$. Let $x_{0}, y_{0} \in V$ satisfy $x_{0}, y_{0} \in \operatorname{ri} D, x_{0} \prec_{G} y_{0}$, and $\left\langle x_{0}, v\right\rangle=\left\langle y_{0}, v\right\rangle$.

Put $x=x_{0}+u+\alpha w$ and $y=y_{0}+u+\beta w$, where $u \in V$ and $\alpha, \beta \in \mathbb{R}$ are such that $u+\alpha w \in \operatorname{span} v$ and $u+\beta w \in \operatorname{span} v$.

Let $F \in \mathcal{S}(A, x, y)$, where $A=$ ri $D+\operatorname{span} v$.

Under the above assumptions, the following three assertions hold.

(A) $F(y)-F(x) \geq(\beta-\alpha) \int_{0}^{1}\langle\nabla F(x+t h), w\rangle d t$.

(B) If $\alpha=\beta$ then $F(x) \leq F(y)$.

(C) If $\alpha \leq \beta$ and $\langle\nabla F(z), w\rangle \geq 0$ for $z \in[x, y]$, then $F(x) \leq F(y)$.

6.3. Extended $G$-majorization. For classical majorization $\prec$ on $\mathbb{R}^{n}$ the following criterions are well-known. 
Theorem 6.10 ([39, Theorem B.1, p. 186], [75. Lemma 4]). Let $x, y \in \mathbb{R}^{n}, y_{1} \geq y_{2} \geq$ $\ldots \geq y_{n}, \sum_{i=1}^{n} x_{i}=\sum_{i=1}^{n} y_{i}$. If there exists $m(1 \leq m \leq n, m \in \mathbb{N})$ such that

$$
y_{i} \leq x_{i} \text { for } i=1,2, \ldots, m \text { and } y_{j} \geq x_{j} \text { for } j=m+1, m+2, \ldots, n,
$$

then

$$
y \prec x .
$$

TheOrem 6.11 ([40, Theorem 2.4]). If $x=\left(x_{1}, x_{2}, \ldots, x_{n}\right)$ and $y=\left(y_{1}, y_{2}, \ldots, y_{n}\right)$ with $x_{i}>0,1 \leq i \leq n$, and $y_{1} \geq y_{2} \geq \ldots \geq y_{n}>0$ and

$$
\frac{x_{1}}{y_{1}} \geq \ldots \geq \frac{x_{n}}{y_{n}}
$$

then

$$
\frac{y}{\sum_{j=1}^{n} y_{j}} \prec \frac{x}{\sum_{j=1}^{n} x_{j}} .
$$

We extend Theorems 6.10 and 6.11 from the classical majorization $\prec$ to group-induced cone orderings.

Theorem 6.12 ([60, Theorem 3.1]). Assume that $\prec_{G}$ is a GIC ordering on $V$ induced by finite group $G$ and convex cone $D_{0}=$ cone $\left\{s_{1}, s_{2}, \ldots, s_{q}\right\}$ with $s_{1}, s_{2}, \ldots, s_{q} \in V$. Set $D=$ cone $\left\{s_{1}, s_{2}, \ldots, s_{n}\right\}$, where $s_{1}, s_{2}, \ldots, s_{n}$ form a basis of $V, n \leq q$.

Let $x, y, v \in V$ with $\langle x, v\rangle y \in D_{0}$ (e.g., $\langle x, v\rangle \geq 0$ and $\left.y \in D_{0}\right)$. Suppose that for $k=1,2, \ldots, n$ there exist dual bases $e=\left(e_{1}, \ldots, e_{n}\right)$ and $d=\left(d_{1}, \ldots, d_{n}\right)$ of $V$, and index sets $J_{1}$ and $J_{2}$ with $J_{1} \cup J_{2}=\{1,2, \ldots, n\}$ such that

(i) $x$ is $\eta$,y-separable on $J_{1}$ and $J_{2}$ w.r.t. $e$ with $\eta=\langle x, v\rangle /\langle y, v\rangle$ and $\langle y, v\rangle>0$,

(ii) $s_{k}$ is v-separable on $J_{1}$ and $J_{2}$ w.r.t. d.

If $n<q$, assume additionally that

$$
\left\langle\langle x, v\rangle y, s_{k}\right\rangle \leq\left\langle\langle y, v\rangle x, s_{k}\right\rangle \quad \text { for } k=n+1, \ldots, q .
$$

Then the following G-majorization inequality holds:

$$
\langle x, v\rangle y \prec_{G}\langle y, v\rangle x .
$$

If $\langle x, v\rangle\langle y, v\rangle>0$ then 46 becomes

$$
\frac{y}{\langle y, v\rangle} \prec_{G} \frac{x}{\langle x, v\rangle} .
$$

Theorem 6.13 ([60, Theorem 3.4]). Assume that $\prec_{G}$ is a GIC ordering on $V$ induced by finite group $G$ and convex cone $D_{0}=$ cone $\left\{s_{1}, s_{2}, \ldots, s_{q}\right\}$ with $s_{1}, s_{2}, \ldots, s_{q} \in V$. Set $D=$ cone $\left\{s_{1}, s_{2}, \ldots, s_{n}\right\}$, where $s_{1}, s_{2}, \ldots, s_{n}$ form a basis of $V, n \leq q$.

Let $x, y, v \in V$ be such that $\langle x, v\rangle>0,\langle y, v\rangle>0$ and $y \in D_{0}$.

Suppose that there exist a basis $e=\left(e_{1}, e_{2}, \ldots, e_{n}\right)$ and an index $m \in\{0,1,2, \ldots, n\}$ such that

(i) $x$ is $\eta, y$-separable on $J_{1}$ and $J_{2}$ w.r.t. e, where $\eta=\frac{\langle x, v\rangle}{\langle y, v\rangle}$ and $J_{1}=\{1,2, \ldots, m\}$, $J_{2}=\{m+1, m+2, \ldots, n\}$,

(ii) the vectors $s_{1}, s_{2}, \ldots, s_{n}, e_{1}, e_{2}, \ldots, e_{n}$ and $v$ satisfy conditions $s_{i} \in$ cone $\left\{e_{1}, \ldots, e_{m}\right\} \quad$ and $\quad v-s_{j} \in$ cone $\left\{e_{m+1}, \ldots, e_{n}\right\} \quad$ for $i \in J_{1}$ and $j \in J_{2}$. 
If $n<q$, assume additionally that 45 holds. Then

$$
\frac{y}{\langle y, v\rangle} \prec_{G} \frac{x}{\langle x, v\rangle} \text {. }
$$

If in addition $\langle x, v\rangle=\langle y, v\rangle$ then

$$
y \prec_{G} x .
$$

Corollary 6.14 ([60, Corollary 3.5]). Under the hypotheses of Theorem 6.13, let conditions (i)-(ii) of Theorem 6.13 be replaced by the following statement:

There exists a basis $e=\left(e_{1}, e_{2}, \ldots, e_{n}\right)$ of $V$ such that

(i) $\frac{\left\langle x, e_{1}\right\rangle}{\left\langle y, e_{1}\right\rangle} \geq \frac{\left\langle x, e_{2}\right\rangle}{\left\langle y, e_{2}\right\rangle} \geq \ldots \geq \frac{\left\langle x, e_{n}\right\rangle}{\left\langle y, e_{n}\right\rangle} \quad$ (with positive denominators),

(ii) the vectors $s_{1}, s_{2}, \ldots, s_{n}, e_{1}, e_{2}, \ldots, e_{n}$ and $v$ satisfy conditions

$$
s_{i}=e_{1}+e_{2}+\ldots+e_{i} \quad \text { for } \quad i=1,2, \ldots, n, \quad \text { and } \quad v=s_{n} .
$$

Then inequality 47) holds.

By comparing (47) and (48) we can call the relation 47) extended $G$-majorization of $y$ and $x$ with respect to $v$.

7. Shi and Ky Fan type inequalities. H.-N. Shi 67] proved the following majorization result (see also [26, Lemmas 2.2-2.3], [39, p. 9], [74, Lemma 2]).

THEOREM 7.1 ([67, pp. 81-83]). If $x=\left(x_{1}, \ldots, x_{n}\right) \in \mathbb{R}^{n}, n \geq 2, \sum_{i=1}^{n} x_{i}=s>0$, $c \geq s$, then

$$
\frac{c v+x}{n c+s} \prec \frac{x}{s} \quad \text { and } \quad \frac{c v-x}{n c-s} \prec \frac{x}{s}
$$

where $v=(1,1, \ldots, 1) \in \mathbb{R}^{n}$.

We denote by $A\left(z_{1}, z_{2}, \ldots, z_{n}\right)$ and $G\left(z_{1}, z_{2}, \ldots, z_{n}\right)$ the arithmetic and geometric means, respectively, of positive numbers $z_{1}, z_{2}, \ldots, z_{n}$.

The following Ky Fan's result is of interest.

TheOREM 7.2 ([5, p. 5]). Let $x_{i} \in(0,1 / 2](i=1,2, \ldots, n)$. Then the following Ky Fan's inequality holds:

$$
\frac{G\left(x_{1}, x_{2}, \ldots, x_{n}\right)}{G\left(1-x_{1}, 1-x_{2}, \ldots, 1-x_{n}\right)} \leq \frac{A\left(x_{1}, x_{2}, \ldots, x_{n}\right)}{A\left(1-x_{1}, 1-x_{2}, \ldots, 1-x_{n}\right)} .
$$

Our purpose in this section is to give an extension of Theorems 7.1 and 7.2 from the classical majorization preorder $\prec$ to a class of group-induced cone orderings $\prec_{G}$. As applications, some Ky Fan type inequalities are established.

7.1. G-majorization inequalities of Shi type. In the forthcoming theorems we provide extensions of the above result of Shi (see also [39, p. 9]).

Theorem 7.3 ([58, Theorem 7]). Let $(V, G, D)$ be an Eaton triple and let $M_{G}(V)=$ $\operatorname{span} v$ for some nonzero $v \in V$. Then

$$
\frac{c v+x}{\langle c v+x, v\rangle} \prec_{G} \frac{x}{\langle x, v\rangle} \quad \text { for } x \in V \text { with }\langle x, v\rangle>0, c \geq 0 \text {. }
$$

We need some notation. 
Definition 7.4. A sequence $a=\left(a_{1}, a_{2}, \ldots, a_{n}\right) \in \mathbb{R}^{n}$ is said to be relatively convex (resp., relatively concave) with respect to sequence $b=\left(b_{1}, b_{2}, \ldots, b_{n}\right) \in \mathbb{R}^{n}$ if

$$
\left|\begin{array}{ccc}
1 & b_{k} & a_{k} \\
1 & b_{l} & a_{l} \\
1 & b_{m} & a_{m}
\end{array}\right| \geq(\leq) 0
$$

whenever $k, l, m \in\{1,2, \ldots, n\}$ and $b_{k} \leq b_{l} \leq b_{m}$ (cf. [44, p. 2]).

Theorem 7.5 ([58, Theorem 11]). Let $(V, G, D)$ be an Eaton triple with $M_{G}(V)=\operatorname{span} v$ for some nonzero $v \in V,\|v\|>1$. Let $D=$ cone $\left\{t_{1}, \ldots, t_{n}, t_{n+1}\right\}$ with $t_{0}=0, t_{n}=v$, $t_{n+1}=-t_{n}$ and $1 \leq\left\langle t_{i}, v\right\rangle \leq\left\langle t_{n}, v\right\rangle$ for $i=1, \ldots, n$, and let $g_{0} \in G$ be such that $g_{0} D=-D$ and $t_{n-j}=v-g_{0}^{-1} t_{j}$ for $j=1,2, \ldots, n$.

Assume that $x \in V$ and $c \in \mathbb{R}$ are such that $c \geq\langle x, v\rangle>0$.

Let $a=\left(a_{0}, a_{1}, \ldots, a_{n}\right)$ and $b=\left(b_{0}, b_{1}, \ldots, b_{n}\right)$, where $a_{i}=\left\langle t_{i}, x_{\downarrow}\right\rangle$ and $b_{i}=\left\langle t_{i}, v\right\rangle$ for $i=0,1,2, \ldots, n$. Suppose that a is relatively concave with respect to $b$. Then

$$
\frac{c v-x}{\langle c v-x, v\rangle} \prec_{G} \frac{x}{\langle x, v\rangle} .
$$

7.2. Generalization of Ky Fan inequality. An application of Theorems 7.3 and 7.5 leads to the following corollaries.

COROllary 7.6 ([58, Corollary 12]). Under the assumptions of Theorem 7.3, let $\varphi$ : $A \rightarrow(0,+\infty)$ be a positively homogeneous $G$-increasing (resp. G-decreasing) function on $G$-invariant set $A \subset V$.

If $x \in A$ and $c \geq 0$ with $\langle x, v\rangle>0$, then

$$
\frac{\langle x, v\rangle}{\langle c v+x, v\rangle} \leq(\geq) \frac{\varphi(x)}{\varphi(c v+x)} .
$$

Corollary 7.7 ([58, Corollary 13]). Under the assumptions of Theorem 7.5, let $\varphi$ : $A \rightarrow(0,+\infty)$ be a positively homogeneous $G$-increasing (resp. $G$-decreasing) function on $G$-invariant set $A \subset V$.

If $x \in A$ and $c \in \mathbb{R}$ with $0<\langle x, v\rangle \leq c$, then

$$
\frac{\langle x, v\rangle}{\langle c v-x, v\rangle} \leq(\geq) \frac{\phi(x)}{\phi(c v-x)} .
$$

See [26, 36, 67, 76] and references therein for a number of results of type [49 - 50 for S-convex or S-concave functions $\varphi$.

ExAmPLE 7.8. Let $V=\mathbb{R}^{n}, G=\mathbb{P}_{n}, D=\mathbb{R}_{\downarrow}^{n}, v=(1,1, \ldots, 1), c=1$ and

$$
\varphi(z)=\left(\prod_{i=1}^{n} z_{i}\right)^{1 / n} \quad \text { for } z=\left(z_{1}, z_{2}, \ldots, z_{n}\right) \in(0,+\infty)^{n} .
$$

Then inequality (49) of Corollary 7.6 leads to the following Xia-Chu's inequality:

$$
\frac{A\left(x_{1}, \ldots, x_{n}\right)}{A\left(1+x_{1}, \ldots, 1+x_{n}\right)} \geq \frac{G\left(x_{1}, \ldots, x_{n}\right)}{G\left(1+x_{1}, \ldots, 1+x_{n}\right)} \quad \text { for } 0<x_{i}, i=1, \ldots, n,
$$

where $A(\cdot)$ and $G(\cdot)$ denote the arithmetic and geometric means of $(\cdot)$ [76, Corollary 4.3]. 
Using (50) of Corollary 7.7 yields the following variant of Ky Fan's inequality:

$$
\frac{A\left(x_{1}, \ldots, x_{n}\right)}{A\left(1-x_{1}, \ldots, 1-x_{n}\right)} \geq \frac{G\left(x_{1}, \ldots, x_{n}\right)}{G\left(1-x_{1}, \ldots, 1-x_{n}\right)}
$$

Whenever $0<\sum_{i=1}^{n} x_{i} \leq 1, i=1, \ldots, n$.

8. Applications to Grüss and Ostrowski type inequalities. We denote by $L_{[a, b]}^{p}$ $(1 \leq p<\infty)$ the space of $p$-power integrable functions on interval $[a, b]$ equipped with the norm

$$
\|f\|_{p}=\left(\int_{a}^{b}|f(t)|^{p} d t\right)^{1 / p} .
$$

The symbol $L_{[a, b]}^{\infty}$ stands for the space of all essentially bounded functions on $[a, b]$ with the norm

$$
\|f\|_{\infty}=\operatorname{essip}_{x \in[a, b]}|f(x)| .
$$

For two real functions $f, g:[a, b] \rightarrow \mathbb{R}$ such that $f, g, f g \in L_{[a, b]}^{1}$, the Chebyshev functional is defined by

$$
T(f, g)=\frac{1}{b-a} \int_{a}^{b} f(t) g(t) d t-\frac{1}{b-a} \int_{a}^{b} f(t) d t \cdot \frac{1}{b-a} \int_{a}^{b} g(t) d t .
$$

Chebyshev [8] proved that if $f^{\prime}, g^{\prime} \in L_{[a, b]}^{\infty}$, then

$$
|T(f, g)| \leq \frac{1}{12}(b-a)^{2}\left\|f^{\prime}\right\|_{\infty}\left\|g^{\prime}\right\|_{\infty} .
$$

Grüss [25] showed that

$$
|T(f, g)| \leq \frac{1}{4}\left(\beta_{0}-\alpha_{0}\right)\left(\delta_{0}-\gamma_{0}\right)
$$

provided two bounded integrable functions $f, g:[a, b] \rightarrow \mathbb{R}$ satisfy

$$
\alpha_{0} \leq f(t) \leq \beta_{0} \quad \text { and } \quad \gamma_{0} \leq g(t) \leq \delta_{0} \quad \text { for } t \in[a, b] .
$$

See [10, 21, 31, 37] for generalizations and extensions of 51.

8.1. Grüss type inequalities. Let $(V,\langle\cdot, \cdot\rangle)$ be an inner product space over $\mathbb{F}=\mathbb{R}$ or $\mathbb{C}$ endowed with the corresponding norm $\|\cdot\|=\langle\cdot, \cdot\rangle^{1 / 2}$. A Grüss type inequality estimates from above the quantity $|\langle x, y\rangle-\langle x, v\rangle\langle v, y\rangle|$ with $x, y, v \in V,\|v\|=1$ (cf. [11, 10, 65]).

Remind that if $K \subset V$ is a convex cone, then $\leq_{K}$ is cone preorder on $V$ defined by

$$
y \leq_{K} x \quad \text { iff } \quad x-y \in K .
$$

THEOREM 8.1 ([56, Theorem 4.2]). Let $(V,\langle\cdot, \cdot\rangle)$ be an inner product space over $\mathbb{F}=\mathbb{R}$ or $\mathbb{C}$, and let $v \in V$ with $\|v\|=1$. Assume that $x, y, \alpha, \beta, \gamma, \delta \in V$ are vectors such that

(a) $\alpha+\beta \in \operatorname{span} v$ and $\gamma+\delta \in \operatorname{span} v$,

(b) $\alpha \leq_{K_{1}} x \leq_{\text {dual } K_{1}} \beta$ and $\gamma \leq_{K_{2}} y \leq_{\text {dual } K_{2}} \delta$ for some convex cones $K_{1}, K_{2} \subset V$.

Then we have the inequality

$$
|\langle x, y\rangle-\langle x, v\rangle\langle v, y\rangle| \leq \frac{1}{4}\|\beta-\alpha\|\|\delta-\gamma\| .
$$


If the vectors $\alpha, \beta, \gamma, \delta$ are proportional to $v$, Theorem 8.1 reduces to [11, Theorem 1].

Remind that a function $\varphi:[a, b] \rightarrow \mathbb{R}$ is said to be a constant function, if there exists a constant $c \in \mathbb{R}$ such that $\varphi(t)=c$ for $t \in[a, b]$.

Corollary 8.2 ([56, Corollary 4.5]). Let $f, g, \alpha, \beta, \gamma, \delta \in L_{[a, b]}^{2}$ be functions such that

(a) $\alpha+\beta$ and $\gamma+\delta$ are constant functions,

(b) $\alpha(t) \leq f(t) \leq \beta(t)$ and $\gamma(t) \leq g(t) \leq \delta(t)$ for $t \in[a, b]$,

or, more generally,

$$
\int_{a}^{b}(\beta(t)-f(t))(f(t)-\alpha(t)) d t \geq 0 \quad \text { and } \quad \int_{a}^{b}(\delta(t)-g(t))(g(t)-\gamma(t)) d t \geq 0 .
$$

Then we have the inequality

$$
|T(f, g)| \leq \frac{1}{4(b-a)}\left(\int_{a}^{b}(\beta(t)-\alpha(t))^{2} d t\right)^{1 / 2}\left(\int_{a}^{b}(\delta(t)-\gamma(t))^{2} d t\right)^{1 / 2} .
$$

For many functions $f$ and $g$ with appropriate choice of functions $\alpha(\cdot), \beta(\cdot), \gamma(\cdot)$ and $\delta(\cdot)$, inequality (52) provides a tighter estimate than (51) (see [56, Example 4.6]).

8.2. Ostrowski-Grüss type inequalities. A related result to (51) is the following Ostrowski's inequality [43, p. 468].

THEOREM 8.3 ([43, p. 468]). If $f:[a, b] \rightarrow \mathbb{R}$ is a differentiable function with bounded derivative, then

$$
\left|f(x)-\frac{1}{b-a} \int_{a}^{b} f(t) d t\right| \leq\left[\frac{1}{4}+\frac{(x-(a+b) / 2)^{2}}{(b-a)^{2}}\right](b-a)\left\|f^{\prime}\right\|_{\infty} \quad \text { for } x \in[a, b] .
$$

Dragomir and Wang [14] showed Ostrowski-Grüss type inequality as follows.

THEOREM 8.4 ([14]). If $f:[a, b] \rightarrow \mathbb{R}$ is a differentiable function with bounded derivative such that

$$
\alpha_{0} \leq f^{\prime}(t) \leq \beta_{0} \quad \text { for } t \in[a, b]
$$

then

$$
\begin{aligned}
\mid f(x)-\frac{1}{b-a} \int_{a}^{b} f(t) d t-\frac{f(b)-f(a)}{b-a}(x & \left.-\frac{a+b}{2}\right) \mid \\
& \leq \frac{1}{4}(b-a)\left(\beta_{0}-\alpha_{0}\right) \quad \text { for } x \in[a, b] .
\end{aligned}
$$

Some improvements of (54) can be found in 9, 41.

Grüss and Ostrowski type inequalities have many applications (see [10, 13, 14, 38, 63, ).

Here we present an extension of Grüss' inequality in $L^{p}$-spaces (cf. [7, Theorems 2 and 3], [12, p. 2]).

Theorem 8.5 ([57, Theorem 2.1]). Let $f, \alpha, \beta \in L_{[a, b]}^{p}$ and $g \in L_{[a, b]}^{q}\left(\frac{1}{p}+\frac{1}{q}=1\right.$, $1 \leq p \leq \infty)$ be functions such that

(a) $\alpha+\beta$ is a constant function, and

(b) $\alpha(t) \leq f(t) \leq \beta(t)$ for $t \in[a, b]$. 
Then we have the inequality

$$
|T(f, g)| \leq \frac{1}{2(b-a)}\|\beta-\alpha\|_{p} \cdot\left\|g-\frac{1}{b-a} \int_{a}^{b} g(t) d t\right\|_{q} .
$$

THEOREM 8.6 ([57, Theorem 2.4]). Let $f: I \rightarrow \mathbb{R}$, where $I \subset \mathbb{R}$ is an interval, be a function differentiable in the interior $\stackrel{\circ}{I}$ of $I$, and let $[a, b] \subset \stackrel{\circ}{I}$. Suppose that $f^{\prime}, \alpha, \beta \in L_{[a, b]}^{p}$ $(1 \leq p \leq \infty)$ are functions such that

(a) $\alpha+\beta$ is a constant function, and

(b) $\alpha(t) \leq f^{\prime}(t) \leq \beta(t)$ for $t \in[a, b]$.

Then for $x \in[a, b]$

$$
\begin{aligned}
&\left|f(x)-\frac{1}{b-a} \int_{a}^{b} f(t) d t-\left(x-\frac{a+b}{2}\right) \frac{f(b)-f(a)}{b-a}\right| \\
& \leq \begin{cases}\frac{1}{4}\|\beta-\alpha\|_{p} \frac{(b-a)^{1 / q}}{(q+1)^{1 / q}} & \text { if } 1 \leq q<\infty, \\
\frac{1}{4}\|\beta-\alpha\|_{1} & \text { if } q=\infty,\end{cases}
\end{aligned}
$$

where $\frac{1}{p}+\frac{1}{q}=1$.

COROLlary 8.7 ([57, Corollary 2.5]). Let $f: I \rightarrow \mathbb{R}$, where $I \subset \mathbb{R}$ is an interval, be a function differentiable in the interior $\stackrel{\circ}{I}$ of $I$, and let $[a, b] \subset \stackrel{\circ}{I}$. Suppose that $\alpha_{0}, \beta_{0} \in \mathbb{R}$ are numbers such that $\alpha_{0} \leq f^{\prime}(t) \leq \beta_{0}$ for $t \in[a, b]$.

Then for $x \in[a, b]$ and $1 \leq q \leq \infty$

$$
\begin{aligned}
\left|f(x)-\frac{1}{b-a} \int_{a}^{b} f(t) d t-\left(x-\frac{a+b}{2}\right) \frac{f(b)-f(a)}{b-a}\right| \\
\leq \begin{cases}\frac{1}{4(q+1)^{1 / q}}\left(\beta_{0}-\alpha_{0}\right)(b-a) & \text { if } 1 \leq q<\infty, \\
\frac{1}{4}\left(\beta_{0}-\alpha_{0}\right)(b-a) & \text { if } q=\infty .\end{cases}
\end{aligned}
$$

The case $q=1$ of Corollary 8.7 yields a result of Cheng [9, Theorem 1.5] with the factor $\frac{1}{8}$ on the right-hand side of (56) (cf. [38, Theorem 3]). If $q=2$ then Corollary 8.7 becomes a result of Matić et al. [41, Theorem 6] with the factor $\frac{1}{4 \sqrt{3}}$ on the right-hand side of (56). Finally, the case $q=\infty$ leads to inequality (54) of Dragomir and Wang [14, Theorem 2.1].

\section{References}

[1] B. J. Andersson, An inequality for convex functions, Nordisk Mat. Tidskr. 6 (1958), 25-26.

[2] S. A. Andersson, M. D. Perlman, Group-invariant analogues of Hadamard's inequality, Linear Algebra Appl. 110 (1988), 91-116.

[3] T. Ando, Majorization, doubly stochastic matrices, and comparison of eigenvalues, Linear Algebra Appl. 118 (1989), 163-248.

[4] T. Ando, Majorization and inequalities in matrix theory, Linear Algebra Appl. 199 (1994), $17-67$.

[5] E. F. Beckenbach, R. Bellman, Inequalities, Ergeb. Math. Grenzgeb. 30, Springer, Berlin, 1961. 
[6] R. Bhatia, Matrix Analysis, Grad. Texts in Math. 169, Springer, New York, 1997.

[7] P. Cerone, S. S. Dragomir, New bounds for the Čebyšev functional, Appl. Math. Lett. 18 (2005), 603-611.

[8] P. L. Chebyshev, Sur les expressions approximatives des intégrales définies par les autres prises entre les mêmes limites, Proc. Math. Soc. Charkov 2 (1882), 93-98.

[9] X.-L. Cheng, Improvement of some Ostrowski-Grüss type inequalities, Comput. Math. Appl. 42 (2001), 109-114.

[10] S. S. Dragomir, A Grüss type discrete inequality in inner product spaces and applications, J. Math. Anal. Appl. 250 (2000), 494-511.

[11] S. S. Dragomir, Some majorisation type discrete inequalities for convex functions, Math. Inequal. Appl. 7 (2004), 207-216.

[12] S. S. Dragomir, Bounds for some perturbed Čebyšev functionals, JIPAM. J. Inequal. Pure Appl. Math. 9 (2008), Article 64, 10 pp.

[13] S. S. Dragomir, A. Sofo, An inequality for monotonic functions generalizing Ostrowski and related results, Comput. Math. Appl. 51 (2006), 497-506.

[14] S. S. Dragomir, S. Wang, An inequality of Ostrowski-Grüss' type and its applications to the estimation of error bounds for some special means and for some numerical quadrature rules, Comput. Math. Appl. 39 (1997), no. 11, 15-20.

[15] M. L. Eaton, On group induced orderings, monotone functions, and convolution theorems, in: Inequalities in Statistics and Probability (Lincoln, Neb., 1982), IMS Lecture Notes Monogr. Ser. 5, Inst. Math. Statist., Hayward, CA, 1984, 13-25.

[16] M. L. Eaton, Group induced orderings with some applications in statistics, CWI Newslett. 16 (1987), 3-31.

[17] M. L. Eaton, Lectures on Topics in Probability Inequalities, CWI Tract 35, Stichting Mathematisch Centrum, Centrum voor Wiskunde en Informatica, Amsterdam, 1987.

[18] M. L. Eaton, M. D. Perlman, Reflection groups, generalized Schur functions, and the geometry of majorization, Ann. Probability 5 (1977), 829-860.

[19] K. Fan, On a theorem of Weyl concerning eigenvalues of linear transformations. I, Proc. Nat. Acad. Sci. U.S.A. 35 (1949), 652-655.

[20] K. Fan, Maximum properties and inequalities for the eigenvalues of completely continuous operators, Proc. Nat. Acad. Sci. U.S.A. 37 (1951), 760-766.

[21] A. M. Fink, A treatise on Grüss' inequality, in: Analytic and Geometric Inequalities and Applications, Math. Appl. 478, Kluwer, Dordrecht, 1999, 93-113.

[22] A. M. Fink, Andersson's inequality, Math. Inequal. Appl. 6 (2003), 241-245.

[23] I. Gavrea, T. Trif, On Ky Fan's inequality, Math. Inequal. Appl. 4 (2001), 223-230.

[24] A. Giovagnoli, H. P. Wynn, G-majorization with applications to matrix orderings, Linear Algebra Appl. 67 (1985), 111-135.

[25] G. Grüss, Über das Maximum des absoluten Betrages von $\frac{1}{b-a} \int_{a}^{b} f(x) g(x) d x-$ $\frac{1}{(b-a)^{2}} \int_{a}^{b} f(x) d x \int_{a}^{b} g(x) d x$, Math. Z. 39 (1935), 215-226.

[26] K. Guan, Schur-convexity of the complete elementary symmetric function, J. Inequal. Appl. 2006, Art. ID 67624, 9 pp.

[27] G. H. Hardy, J. E. Littlewood, G. Pólya, Inequalities, Cambridge Univ. Press, Cambridge, 1952.

[28] R. A. Horn, C. R. Johnson, Matrix Analysis, Cambridge Univ. Press, Cambridge, 1985.

[29] R. A. Horn, C. R. Johnson, Topics in Matrix Analysis, Cambridge Univ. Press, Cambridge, 1991. 
[30] J. E. Humphreys, Reflection groups and Coxeter groups, Cambridge Stud. Adv. Math. 29, Cambridge Univ. Press, Cambridge, 1990.

[31] S. Izumino, J. E. Pečarić, B. Tepeš, A Grüss-type inequality and its applications, J. Inequal. Appl. 2005, no. 3, 277-288.

[32] C. R. Johnson, P. M. Nylen, Converse to a singular value inequality common to the Hadamard and conventional products, Linear and Multilinear Algebra 27 (1990), 167-187.

[33] J. Karro, C. K. Li, A unified elementary approach to canonical forms of matrices, SIAM Rev. 39 (1997), 305-309.

[34] A. S. Lewis, Group invariance and convex matrix analysis, SIAM J. Matrix Anal. Appl. 17 (1996), 927-949.

[35] A. S. Lewis, Convex analysis on Cartan subspaces, Nonlinear Anal. 42 (2000), 813-820.

[36] A.-J. Li, X.-M. Wang, C.-P. Chen, Generalizations of the Ky Fan inequality, JIPAM. J. Inequal. Pure Appl. Math. 7 (2006), Article 130, 6 pp.

[37] X. Li, R. N. Mohapatra, R. S. Rodriguez, Grüss-type inequalities, J. Math. Anal. Appl. 267 (2002), 434-443.

[38] Z. Liu, Some Ostrowski-Grüss type inequalities and applications, Comput. Math. Appl. 53 (2007), 73-79.

[39] A. W. Marshall, I. Olkin, B. C. Arnold, Inequalities: Theory of Majorization and its Applications, Springer Ser. Statist., Springer, New York, 2011.

[40] A. W. Marshall, I. Olkin, F. Proschan, Monotonicity of ratios of means and other applications of majorization, in: Inequalities (Ohio, 1965), Academic Press, New York, 1967, 177-190.

[41] M. Matić, J. Pečarić, N. Ujević, Improvement and further generalization of inequalities of Ostrowski-Grüss type, Comput. Math. Appl. 39 (2000), 161-175.

[42] H. F. Miranda, R. C. Thompson, Group majorization, the convex hulls of sets of matrices, and the diagonal elements - singular values inequalities, Linear Algebra Appl. 199 (1994), 131-141.

[43] D. S. Mitrinović, J. E. Pečarić, A. M. Fink, Inequalities Involving Functions and their Integrals and Derivatives, Math. Appl. (East European Ser.) 53, Kluwer Academic, Dordrecht, 1991.

[44] C. P. Niculescu, F. Popovici, The extension of majorization inequalities within the framework of relative convexity, JIPAM. J. Inequal. Pure Appl. Math. 7 (2006), Article 27, 6 pp.

[45] M. Niezgoda, Group majorization and Schur type inequalities, Linear Algebra Appl. 268 (1998), 9-30.

[46] M. Niezgoda, On Schur-Ostrowski type theorems for group majorizations, J. Convex Anal. 5 (1998), 81-105.

[47] M. Niezgoda, G-majorization inequalities for linear maps, Linear Algebra Appl. 292 (1999), 207-231.

[48] M. Niezgoda, Bifractional inequalities and convex cones, Discrete Math. 306 (2006), 231-243.

[49] M. Niezgoda, On linear preservers of normal maps, Linear Algebra Appl. 426 (2007), 149-158.

[50] M. Niezgoda, On the Chebyshev functional, Math. Inequal. Appl. 10 (2007), 535-546.

[51] M. Niezgoda, G-majorization inequalities and canonical forms of matrices, J. Convex Anal. 14 (2007), 35-48. 
[52] M. Niezgoda, G-majorization inequalities for linear maps II, Linear Multilinear Algebra 55 (2007), 1-17.

[53] M. Niezgoda, Some remarks on normal maps with applications to eigenvalues and singular values of matrices, Linear Algebra Appl. 428 (2008), 2116-2129.

[54] M. Niezgoda, Remarks on convex functions and separable sequences, Discrete Math. 308 (2008), 1765-1773.

[55] M. Niezgoda, Morphisms of normal decomposition systems, J. Convex Anal. 16 (2009), 617-632.

[56] M. Niezgoda, Translation-invariant maps and applications, J. Math. Anal. Appl. 354 (2009), 111-124.

[57] M. Niezgoda, A new inequality of Ostrowski-Grüss type and applications to some numerical quadrature rules, Comput. Math. Appl. 58 (2009), 589-596.

[58] M. Niezgoda, Majorization and relative concavity, Linear Algebra Appl. 434 (2011), 19681980.

[59] M. Niezgoda, Schur-Ostrowski type theorems revisited, J. Math. Anal. Appl. 381 (2011), 935-946.

[60] M. Niezgoda, Cone orderings, group majorizations and similarly separable vectors, Linear Algebra Appl. 436 (2012), 579-594.

[61] M. Niezgoda, J. Pečarić, Some remarks on the Chebyshev functional, J. Math. Anal. Appl. 346 (2008), 425-431.

[62] A. Ostrowski, Sur quelques applications des fonctions convexes et concaves au sens de I. Schur, J. Math. Pures Appl. (9) 31 (1952), 253-292.

[63] C. E. M. Pearce, J. Pečarić, N. Ujević, S. Varošanec, Generalizations of some inequalities of Ostrowski-Grüss type, Math. Inequal. Appl. 3 (2000), 25-34.

[64] R. Rado, An inequality, J. London Math. Soc. 27 (1952), 1-6.

[65] P. F. Renaud, A matrix formulation of Grüss inequality, Linear Algebra Appl. 335 (2001), 95-100.

[66] I. Schur, Über eine Klasse von Mittelbildungen mit Anwendungen auf die Determinantentheorie, Sitzber. Berliner Math. Ges. 22 (1923), 9-20.

[67] H.-N. Shi, Refinement and generalization of a class of inequalities for symmetric functions, Math. Practice Theory 29 (1999), 81-84.

[68] A. G. M. Steerneman, G-majorization, group-induced cone orderings and reflection groups, Linear Algebra Appl. 127 (1990), 107-119.

[69] T.-Y. Tam, Partial superdiagonal elements and the singular values of a complex skew symmetric matrix, SIAM J. Matrix Anal. Appl. 19 (1998), 737-754.

[70] T.-Y. Tam, An extension of a result of Lewis, Electron. J. Linear Algebra 5 (1999), 1-10.

[71] T.-Y. Tam, Group majorization, Eaton triples and numerical range, Linear and Multilinear Algebra 47 (2000), 11-28.

[72] C. M. Theobald, An inequality for the trace of the product of two symmetric matrices, Math. Proc. Cambridge Philos. Soc. 77 (1975), 265-267.

[73] Gh. Toader, On Chebyshev's inequality for sequences, Discrete Math. 161 (1996), 317-322.

[74] S. Wu, Generalization and sharpness of the power means inequality and their applications, J. Math. Anal. Appl. 312 (2005), 637-652.

[75] S. Wu, L. Debnath, Inequalities for convex sequences and their applications, Comput. Math. Appl. 54 (2007), 525-534.

[76] W.-F. Xia, Y.-M. Chu, Schur-convexity for a class of symmetric functions and its applications, J. Inequal. Appl. 2009, Art. ID 493759, 15 pp. 